\title{
Sub-TeV singlet scalar dark matter and electroweak vacuum stability with vectorlike fermions
}

\author{
Debasish Borah, ${ }^{*}$ Rishav Roshan $\odot,^{\dagger}$ and Arunansu Silø \\ Department of Physics, Indian Institute of Technology Guwahati, Assam 781039, India
}

(Received 5 August 2020; accepted 6 October 2020; published 26 October 2020)

\begin{abstract}
We study a scalar singlet dark matter (DM) having mass in the sub-TeV regime by extending the minimal scalar singlet DM setup by additional vectorlike fermions. While the minimal scalar singlet DM satisfies the relic and direct detection constraints for mass beyond $\mathrm{TeV}$ only, presence of its portal coupling with vectorlike fermions opens up additional coannihilation channels. These vectorlike fermions also help in achieving electroweak vacuum stability all the way up to Planck scale. We find that for one generation of vectorlike quarks consisting of a $S U(2)_{L}$ doublet and a singlet, scalar singlet DM with mass a few hundred $\mathrm{GeV}$ can indeed satisfy relic, direct detection and other relevant constraints while also making the electroweak vacuum absolutely stable. The same can be achieved by introducing vectorlike leptons too, but with three generations. While the model with vectorlike quarks is minimal, the three generations of vectorlike lepton doublet and neutral singlet can also give rise to light neutrino mass at one loop level.
\end{abstract}

DOI: $10.1103 /$ PhysRevD.102.075034

\section{INTRODUCTION}

Understanding the nature of the dark matter (DM) remains an outstanding problem of present day particle physics. Although there has been significant evidence from astrophysics suggesting the presence of this nonbaryonic, nonluminous and collisionless form of matter in the Universe, their direct evidence is still awaited. Information about its abundance has been obtained from WMAP [1] and PLANCK satellite experiments indicating $\Omega_{\mathrm{DM}} h^{2}=0.120 \pm 0.001 \quad$ [2] at $68 \%$ C.L. with $h=$ Hubble parameter $/\left(100 \mathrm{~km} \mathrm{~s}^{-1} \mathrm{Mpc}^{-1}\right)$ which effectively corresponds to around $26 \%$ of the present Universe's energy density. Due to the lack of knowledge about its nature of interaction, apart from the gravitational one, a plethora of possibilities of DM model building has taken place over the years. Among them, the weakly interacting massive particle (WIMP) paradigm [3] is the most widely studied one. In such a scenario a particle DM candidate, typically having electroweak scale mass and interactions, is produced thermally in the early Universe followed by freeze-out from the bath and hence leaves a relic very close to the observed DM abundance.

\footnotetext{
*dborah@iitg.ac.in

†rishav.roshan@iitg.ac.in

*asil@iitg.ac.in
}

Published by the American Physical Society under the terms of the Creative Commons Attribution 4.0 International license. Further distribution of this work must maintain attribution to the author(s) and the published article's title, journal citation, and DOI. Funded by SCOAP ${ }^{3}$.
Perhaps the most economical realization of such a WIMP scenario is the extension of the standard model (SM) by a scalar singlet field having Higgs portal interaction, odd under an unbroken $Z_{2}$ symmetry [4-6] and hence explaining the stability of DM, a recent update on which can be found in [7]. The scenario is tightly constrained by the relic abundance and direct detection (DD) searches. In fact, present direct detection experiments such as LUX [8], PandaX-II $[9,10]$ and XEXON1T [11,12] allow such a DM candidate beyond $1 \mathrm{TeV}$ only (except near the SM Higgs resonance region). On the other hand, the large hadron collider (LHC) bound on Higgs invisible decay width sets constraints on such a possibility even for lighter DM mass: $m_{\text {DM }}<62.5 \mathrm{GeV}[7,13]$. The latest measurements by the ATLAS collaboration constrains the Higgs invisible decay branching ratio to be below 13\% [13] tightly constraining the coupling of scalar DM with SM Higgs for this low mass range. Hence a significant range of DM mass in this simplest framework is presently excluded which could otherwise be an interesting region for several DM (direct and indirect) and collider experiments.

Apart from explaining the DM, such a singlet scalar extension of the SM can also be useful in keeping the electroweak (EW) vacuum absolutely stable all the way until Planck scale for $m_{\mathrm{DM}}$ above $1 \mathrm{TeV}$ [14]. As it is known that the SM Higgs quartic coupling $\lambda_{H}$ becomes negative at an intermediate scale $\Lambda_{I}^{\mathrm{SM}} \sim 10^{9-10} \mathrm{GeV}$ (depending on the precise value of the top quark mass) according to the renormalization group (RG) evolution, it indicates a possible instability of the Higgs vacuum. Although with the current measured mass of the top quark (central value) $\sim 173.2 \mathrm{GeV}$, the $\mathrm{EW}$ vacuum in the SM 
remains metastable [15-24], a more precise measurement of top quark mass may change the conclusion. In addition, such a metastability of the EW vacuum may not be a welcome feature in the context of primordial inflation [25] (and references therein). This situation would change in the presence of the scalar singlet DM as the negative fermionic contribution (primarily due to top quark) to the RG evolution of $\lambda_{H}$ can now be compensated by the additional Higgs portal interaction of the new scalar.

Study of such scalar singlet DM has been extended in different directions where additional portal couplings of the scalar are engaged on top of the usual Higgs portal interaction. One such possibility is to include DM portal couplings with new vectorlike leptons [26-32] and quarks [33-37]. The interesting feature that came out of these studies is the presence of radiative corrections that not only affects the DM annihilations significantly but also leads to interesting observations for DM indirect searches. Such studies are also relevant for collider searches. Another extension of the minimal singlet scalar DM model involves additional scalar with nonzero vacuum expectation value (VEV) as done in [38]. The purpose of the work was to show that in the presence of such a scalar, the low-mass window (below $500 \mathrm{GeV}$ ) for the DM can be reopened. The second scalar is shown to also help in making the EW vacuum stable even when large neutrino Yukawa coupling is present.

In this work, we include additional $Z_{2}$ odd vectorlike fermions (VLFs): first with vectorlike quarks (VLQs) and then with vectorlike leptons (VLLs) such that the scalar DM can coannihilate with these additional fermions. This contribution affecting the relic abundance (to some extent) gets decoupled from the ones giving rise to tree level spin independent DM-nucleon scattering and hence DM with mass below $1 \mathrm{TeV}$ is expected to be revived. Although phenomenological implications of such a setup have already been addressed elsewhere from different perspectives [26-37], here we have one more motivation and that is to ensure the stability of electroweak vacuum all the way up to the Planck scale. The idea emerges from a recent observation [39] that, though we know fermions coupling to the Higgs contribute negatively to the RG running of quartic coupling $\lambda_{H}$ in general, the presence of VLQs can actually affect the $S U(3)$ gauge coupling in such a way which in turn is useful in keeping $\lambda_{H}$ positive at all scales. We show that the same idea can be extended to VLLs also which affect the $S U(2)$ gauge coupling in a similar way to keep $\lambda_{H}$ positive at all scales, though more generations of doublet-singlet VLLs are required to include.

Motivated by the possibility of having a common framework for stable electroweak vacuum and a sub-TeV scalar singlet DM, we first consider an extension of SM by a dark sector consisting of a real scalar singlet DM and two types of VLQs (all $Z_{2}$ odd) transforming as doublet and singlet under $S U(2)_{L}$ gauge symmetry of the SM. We show the new available regions of DM parameter space with mass below a $\mathrm{TeV}$ allowed from all relevant constraints from cosmology and direct searches. We also show that for the same DM parameter space, the electroweak vacuum stability criteria can be satisfied. In the latter half of this paper, we study a similar model but with one vectorlike lepton doublet and a neutral singlet per generation, both odd under the $Z_{2}$ symmetry. We find that at least three such generations of leptons are required to achieve absolute electroweak vacuum stability with the help of DM-Higgs portal coupling (to some extent) where a sub-TeV scalar singlet DM becomes allowed from all phenomenological constraints. While this scenario requires more additional fermions compared to the VLQ model, the additional leptons can also take part in generating light neutrino mass at one loop level.

This paper is organized as follows. In Sec. II, we describe particle content of our model with VLQs and DM, the relevant interactions, particle spectrum and existing constraints. In Sec. III, we summarize the constraints applicable to this scenario. Then in Sec. IV, we first discuss the DM phenomenology for the model with VLQs followed by the issues related to electroweak vacuum stability in V. A combined analysis and parameter space are then presented in Sec. VI. In Sec. VII, we replace the VLQs by VLLs and analyze the DM and vacuum stability in detail which is inclusive of constraints applicable to the scenario, allowed parameter space and a relative comparison between the two scenarios considered in this work. Finally we conclude in Sec. VIII. Appendices A and B are provided to summarize the beta functions of the model parameters.

\section{THE MODEL WITH VLQ}

As stated in the Introduction, we extend the SM fermionfields content by two types of vectorlike quarks [being triplet under $S U(3)_{c}$ ]: a $S U(2)_{L}$ doublet $\mathcal{F}^{T}=\left(\mathcal{F}_{1}^{\prime} \mathcal{F}_{2}\right)$, and singlet $f^{\prime}$ VLQs. In addition, a SM singlet real scalar field $S$ is included which would play the role of DM. These beyond the standard model (BSM) fields are odd under the $Z_{2}$ symmetry while all SM fields are even under it. This unbroken $Z_{2}$ symmetry guarantees the stability of the DM, as usual. The fermion and scalar content of the model inclusive of the SM ones and their respective charges are shown in Table I. The Lagrangian terms, invariant under the symmetries considered, involving Yukawa interactions between the BSM or dark sector fields of our framework and the SM fields can now be written as

$$
\begin{aligned}
-\mathcal{L}^{\mathrm{VLQ}}= & M_{\mathcal{F}} \overline{\mathcal{F}} \mathcal{F}+M_{f} \bar{f}^{\prime} f^{\prime}+y \overline{\mathcal{F}} \tilde{H} f^{\prime} \\
& +\alpha_{1} \overline{\mathcal{F}}_{R} S Q_{L}+\alpha_{2} \bar{f}_{L}^{\prime} S u_{R}+\text { H.c. }
\end{aligned}
$$

Here $L, R$ denote the left- and right-handed projections respectively. Note that the choice of hypercharges of the VLQs $\left(Y_{F}, Y_{f^{\prime}}\right)$ is guided by the consideration that they are 
TABLE I. Particles and their charges under different symmetries.

\begin{tabular}{lcccc}
\hline \hline Particle & $S U(3)_{c}$ & $S U(2)_{L}$ & $U(1)_{Y}$ & $Z_{2}$ \\
\hline$Q_{L}$ & 3 & 2 & $\frac{1}{6}$ & +1 \\
$u_{R}$ & 3 & 1 & $\frac{2}{3}$ & +1 \\
$d_{R}$ & 3 & 1 & $-\frac{1}{3}$ & +1 \\
$l_{L}$ & 1 & 2 & $-\frac{1}{2}$ & +1 \\
$e_{R}$ & 1 & 1 & -1 & +1 \\
$H$ & 1 & 2 & $\frac{1}{2}$ & +1 \\
$S$ & 1 & 1 & 0 & -1 \\
$\mathcal{F}$ & 3 & 2 & $\frac{1}{6}$ & -1 \\
$f^{\prime}$ & 3 & 1 & $\frac{2}{3}$ & -1 \\
\hline \hline
\end{tabular}

SM-like. For the sake of minimality, we choose the singlet VLQ to be of up type only, considering it to be down type though would not have a significant impact on our results and conclusion. The bare masses of $\mathcal{F}$ and $f^{\prime}$ are indicated by $M_{\mathcal{F}}$ and $M_{f}$ respectively.

Turning into the scalar part of the Lagrangian, the most general renormalizable scalar potential of our model, $V(H, S)$ can be divided into three parts: (i) $V_{H}$ : sole contribution of the SM Higgs $H$; (ii) $V_{S}$ : individual contribution of the scalar singlet $S$; and (iii) $V_{\text {int }}$ : interaction among $H$ and $S$ fields, as

$$
V(H, S)=V_{H}+V_{S}+V_{\mathrm{int}},
$$

where

$V_{H}=-\mu_{H}^{2} H^{\dagger} H+\lambda_{H}\left(H^{\dagger} H\right)^{2} ; \quad V_{S}=\frac{1}{2} M_{S}^{2} S^{2}+\frac{\lambda_{S}}{4 !} S^{4}$,

$$
\text { and } \quad V_{\mathrm{int}}=\frac{\lambda_{H S}}{2}\left(H^{\dagger} H\right) S^{2} \text {. }
$$

Once the electroweak symmetry breaking (EWSB) takes place with $v=246 \mathrm{GeV}$ as the SM Higgs VEV, masses of the two physical states, the SM Higgs boson $h$ and $S$, are found to be

$$
m_{h}^{2}=2 \lambda_{H} v^{2}, \quad \text { and } \quad m_{S}^{2}=M_{S}^{2}+\frac{\lambda_{H S}}{2} v^{2} .
$$

Note that the EWSB gives rise to a mixing among the uptype VLQs of the framework. In the basis $\left(\mathcal{F}_{1}^{\prime}, f^{\prime}\right)$, the mass matrix is given by

$$
\mathcal{M}_{\mathrm{VLQ}}=\left(\begin{array}{cc}
M_{\mathcal{F}} & \frac{y v}{\sqrt{2}} \\
\frac{y v}{\sqrt{2}} & M_{f}
\end{array}\right) .
$$

Diagonalizing the above matrix, we get the mass eigenvalues as

$m_{2,1}=\frac{1}{2}\left[M_{\mathcal{F}}+M_{f} \pm \sqrt{\left(M_{\mathcal{F}}+M_{f}\right)^{2}-4\left(M_{\mathcal{F}} M_{f}-\frac{y^{2} v^{2}}{2}\right)}\right.$,

corresponding to the mass eigenstates $f$ and $\mathcal{F}_{1}$. We follow the hierarchy $m_{1}<m_{2}$. These mass eigenstates are related to the flavor eigenstates $\mathcal{F}_{1}^{\prime}, f^{\prime}$ via the mixing angle $\theta$ as

$$
\left(\begin{array}{c}
\mathcal{F}_{1}^{\prime} \\
f^{\prime}
\end{array}\right)=\left(\begin{array}{cc}
c_{\theta} & s_{\theta} \\
-s_{\theta} & c_{\theta}
\end{array}\right)\left(\begin{array}{c}
\mathcal{F}_{1} \\
f
\end{array}\right)
$$

where

$$
\tan 2 \theta=\frac{\sqrt{2} y v}{M_{f}-M_{\mathcal{F}}}
$$

In the subsequent part of the analysis, we choose the physical masses $m_{1}, m_{2}$ and mixing angle $\theta$ as independent variables. Obviously, the other model parameters can be expressed in terms of these independent parameters such as

$$
\begin{gathered}
M_{\mathcal{F}}=m_{1} c_{\theta}^{2}+m_{2} s_{\theta}^{2}, \\
M_{f}=m_{1} s_{\theta}^{2}+m_{2} c_{\theta}^{2}, \\
y=\frac{\sqrt{2}\left(m_{2}-m_{1}\right) c_{\theta} s_{\theta}}{v}=\frac{\sqrt{2}}{v} \Delta_{21} c_{\theta} s_{\theta} .
\end{gathered}
$$

Hence a small $\theta$ would indicate that the lightest eigenstate contains mostly the doublet component.

\section{CONSTRAINTS ON THE MODEL}

Here we summarize all sorts of constraints (theoretical as well as experimental) to be applicable to the model parameters.

\section{A. Theoretical constraints}

(i) Stability: The scalar potential should be bounded from below in all the field directions of the field space. This leads to the following constraints involving the quartic couplings as

$$
\begin{gathered}
\lambda_{H}(\mu), \quad \lambda_{S}(\mu) \geq 0, \\
\lambda_{H S}(\mu)+\sqrt{\frac{2}{3} \lambda_{H}(\mu) \lambda_{S}(\mu)} \geq 0,
\end{gathered}
$$

where $\mu$ is the running scale. These conditions should be analyzed at all the energy scales up to 
the Planck scale $\left(M_{\mathrm{Pl}}\right)$ in order to maintain the stability of the scalar potential until $M_{\mathrm{Pl}}$.

(ii) Perturbativity: A perturbative theory demands that the model parameters should obey

$$
\left|\lambda_{i}\right|<4 \pi \quad \text { and }\left|g_{i}\right|,\left|y, \alpha_{1}, \alpha_{2}\right|<\sqrt{4 \pi} \text {, }
$$

where $g_{i}$ and $\left(y, \alpha_{1}, \alpha_{2}\right)$ are the SM gauge couplings and Yukawa couplings involving BSM fields respectively. We will ensure the perturbativity of the couplings present in the model until the $M_{\mathrm{Pl}}$ by employing the renormalization group equations. In addition, the perturbative unitarity associated with the $\mathrm{S}$ matrix corresponding to $2 \rightarrow 2$ scattering processes involving all two-particle initial and final states $[40,41]$ are considered. It turns out that some of the scalar couplings of Eq. (2) are bounded by

$$
\begin{aligned}
& \lambda_{H}<4 \pi, \lambda_{H S}<8 \pi \text { and } \\
& \frac{1}{4}\left(12 \lambda_{H}+\lambda_{S} \pm \sqrt{16 \lambda_{H S}^{2}+\left(\lambda_{S}-12 \lambda_{H}\right)^{2}}\right)<8 \pi .
\end{aligned}
$$

\section{B. Experimental constraints}

(i) Relic density and direct detection of DM: In order to constrain the parameter space of the model, we use the measured value of the DM relic abundance provided by the Planck experiment [2] and apply the limits on DM direct detection cross section from the LUX [8], PandaX-II [9,10] and XEXON1T $[11,12]$ experiments. Detailed discussion on the dark matter phenomenology involving effects of these constraints is presented in Sec. IV.

(ii) Collider constraints: With the inclusion of additional colored particles (VLQs) in the present setup, the collider searches can be important. In general, the VLQs couple (i) with the SM gauge bosons and (ii) to the SM quarks through Higgs via Yukawatype interactions if allowed by the symmetries of the construction. Such a mixing, after electroweak symmetry breaking, between the VLQs (generically denoted by $T$ say) and SM quarks (say $q$ ) induces a coupling involving $T q X$, with $X \equiv$ gauge bosons or Higgs. This new interaction and mixing with the SM quarks open up various production as well as the decay modes of these VLQs at LHC which in turn provide stringent constraints on their masses and their couplings. In fact, from the $p p$ collision at LHC, VLQs can be pair produced at LHC by strong, weak and electromagnetic interaction (as appropriate). It can also be singly produced via interactions $T q X$, in association with the gauge boson or SM quark in the final state. The dominant production channel depends crucially upon with which generation(s) of SM quarks the VLQs are allowed to couple.

Once produced, each VLQ decays to a SM quark and a gauge boson or Higgs. Such a decay resulting a large number of jets only, cannot be traced back efficiently as the VLQ-origin due to immense QCD background already present in $p p$ collision. However, note that in case $W$ bosons are expected to be present in the final state either from the decay of VLQ or from a subsequent decay of a $t$ quark, VLQ decays lead to the most interesting signature at LHC with transverse missing energy $\left(\mathbb{E}_{T}\right)$, one lepton and jets (VLQs are pair produced). A recent detailed study in this direction [42] shows that VLQ mass below $\sim 1.5 \mathrm{TeV}$ is almost ruled out with $13 \mathrm{TeV}$ LHC data.

However, the above constraint on the mass of the VLQ becomes less stringent in case the VLQ does not have a direct Yukawa coupling with the SM quarks via Higgs as in point (ii) above. Such a situation can be obtained once the VLQ is charged under some discrete symmetry (for example odd under an unbroken $Z_{2}$ as in our case) while SM particles are not. Obviously this significantly reduces the number of production and decay channels of the VLQ due to the absence of VLQ-SM quark mixing. Usually such a construction is related to the presence of a scalar singlet DM (say $S$ as in our case), nontrivially charged under the same discrete symmetry as in [33-36]. In that case, a new portal coupling involving VLQ, scalar singlet and the SM quarks is expected though. Then the decay of VLQs would proceed through the missing energy signal along with the jets provided enough phase space is available. A recent study of this kind of model (topphillic, i.e., VLQ being singlet couples with $S$ and $t_{R}$ ) with LHC run-2 data [36] suggests that the VLQ masses below $1 \mathrm{TeV}$ are excluded only if the DM mass is light enough compared to the VLQ to allow such a decay to happen, e.g., $m_{\mathrm{VLQ}}-m_{S} \gg m_{t}$. On the contrary, if the mass difference falls below $m_{t}$, the constraint becomes much weaker and it is shown to allow $m_{S}$ above $350 \mathrm{GeV}$ with VLQ masses above $\sim 400 \mathrm{GeV}$ [36] or so.

The other possibility where a VLQ couples to lighter SM quark through the scalar singlet DM is extensively investigated in [33], where it is shown that the jets arising from the decay of the VLQs are too soft to be detected at ATLAS or CMS if the VLQs couple to the lighter SM quarks. In that case, inclusion of one or more hard jets (due to radiation of gluons from initial/final/intermediate state) is necessary for the detection of the events. Hence multijets $+\mathscr{E}_{T}$ can provide more practical constraints 
than those obtained from the lowest order two-jet + $E_{T}$ signals. Applying the combined results of multijets $+\mathbb{E}_{T}$ at ATLAS searches together with the constraints coming from the DM direct detection as well as the indirect detection experiments using $\gamma$-rays, the study in [33] allows real scalar singlet DM mass above $300 \mathrm{GeV}$ with $m_{\mathrm{VLQ}} / m_{S} \simeq 1.2$ indicative of VLQ mass $\sim 400 \mathrm{GeV}$. Also for $m_{S}=500 \mathrm{GeV}$, VLQ having mass $550-750 \mathrm{GeV}$ turns out to be in the allowed range.

Note that the above correlation between mass of the VLQ and $m_{S}$ is primarily due to the presence of large Yukawa coupling between VLQs, DM and the SM quark which is also required to satisfy the DM relic density in the work of [33]. Instead in our case, it turns out that this particular coupling plays only a subdominant role in dark matter phenomenology. We have the freedom of choosing smaller Yukawa coupling (compared to [33]) as in our case the correct relic is effectively produced by the annihilation of VLQs (note that we have both the singlet and doublet VLQs) and the Yukawa coupling $\alpha$ can be restricted to be small. Hence limits obtained on $m_{1} / m_{S}$ in [33] do not apply here. On the other hand, we work with relatively lighter VLQ, we maintain the mass splitting between the lightest VLQ and the DM to be smaller than $m_{t}$ so that constraints from the VLQ decay associated with jets $+\mathbb{E}_{T}+$ charged lepton would not be applicable here. Even then, we use the VLQ's mass around $500 \mathrm{GeV}$ as a conservative consideration for their mass. A weaker bound on VLQ's mass however prevails as $m_{\mathrm{VLQ}}>100 \mathrm{GeV}$ similar to the constraints in the case of squark searches, from the LEP experiment [43].

\section{DARK MATTER PHENOMENOLOGY}

In this section, we elaborate on the strategy to calculate the relic density and the direct detection of the dark matter $S$ in the present setup. As we have discussed, apart from the scalar singlet $S$ the present setup also incorporates additional fermions, in the form of one vectorlike quark doublet $\mathcal{F}$ and one vectorlike quark singlet $f^{\prime}$. These VLQs talk to the SM via its (i) Yukawa interactions with (a) the dark matter $S$, (b) the SM Higgs and (ii) gauge interaction (e.g., with gluons). Below we provide a list of relevant Yukawa interaction vertices [belonging to (i) as above].

$$
\begin{aligned}
S \overline{\mathcal{F}}_{1} u: & \frac{1}{2}\left[\alpha_{1} c_{\theta}\left(1-\gamma_{5}\right)-\alpha_{2} s_{\theta}\left(1+\gamma_{5}\right)\right], \\
S \overline{\mathcal{F}}_{2} d: & \frac{1}{2}\left[\alpha_{1}\left(1-\gamma_{5}\right)\right], \\
S \bar{f} u: & \frac{1}{2}\left[\alpha_{1} s_{\theta}\left(1-\gamma_{5}\right)+\alpha_{2} c_{\theta}\left(1+\gamma_{5}\right)\right], \quad \text { and }
\end{aligned}
$$

$$
\begin{gathered}
h \overline{\mathcal{F}}_{1} f: \frac{y}{\sqrt{2}}\left(1-2 s_{\theta}^{2}\right), \\
h \overline{\mathcal{F}}_{1} \mathcal{F}_{1}:-\frac{y}{\sqrt{2}} c_{\theta} s_{\theta}, \\
h \bar{f} f: \frac{y}{\sqrt{2}} c_{\theta} s_{\theta},
\end{gathered}
$$

where $u$ represents all the up-type SM quarks and $d$ represents all the down-type SM quarks.

The presence of these additional interactions opens up new possibilities for the singlet scalar dark matter to coannihilate with the new VLQs into the SM final states. Now, in order to study the evolution of the DM in the Universe one needs to solve the Boltzmann equation of the DM. Before going into the details of the Boltzmann equation, we first identify and categorize different annihilation and coannihilation channels of the dark matter $S$. In Fig. 1 we show all the possible annihilation channels of the dark matter, whereas in Fig. 2 we show only the additional coannihilation channels which come into the picture due to the presence of additional fermions discussed above, and finally in Fig. 3 we show all the possible annihilation channels of the VLQs in the present setup. Considering the DM $S$ as the lightest field among the $Z_{2}$ odd ones, diagrams involving VLQs in the final state will not contribute in the annihilation process.

\section{A. Methodology}

As discussed above, the coannihilation of the dark matter $S$ with the VLQs would play a crucial role in explaining the relic density. It has been also shown in [33-37] that the annihilation among the VLQs themselves can give a significant contribution towards the relic density of the DM. The relic density of the DM with mass $m_{S}$ is given by [5]

$$
\Omega h^{2}=\frac{1.09 \times 10^{9} \mathrm{GeV}^{-1}}{g_{*}^{1 / 2} M_{\mathrm{Pl}}} \frac{1}{J\left(x_{f}\right)},
$$

where $J\left(x_{f}\right)$ is given by

$$
J\left(x_{f}\right)=\int_{x_{f}}^{\infty} \frac{\langle\sigma|v|\rangle_{\text {eff }}}{x^{2}} \mathrm{dx} .
$$

$\langle\sigma|v|\rangle_{\text {eff }}$ in Eq. (17) is the effective thermal average DM annihilation cross sections including contributions from the coannihilations and is given by 


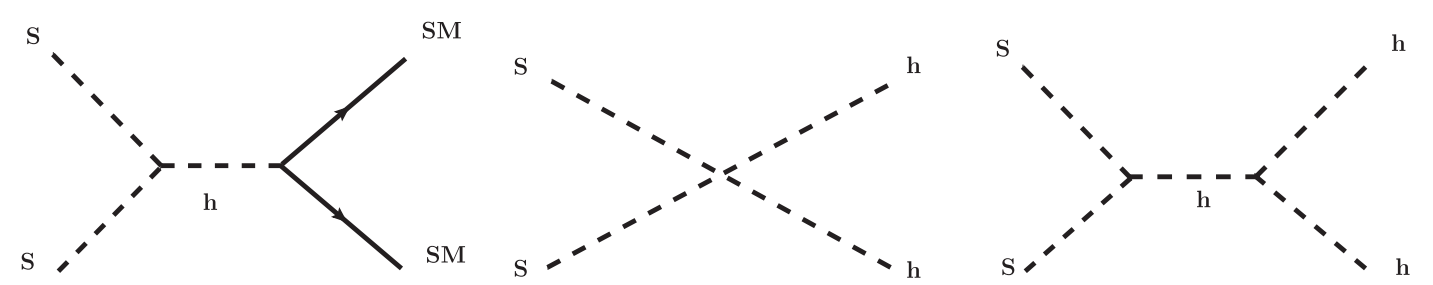

(a)

(b)

(c)

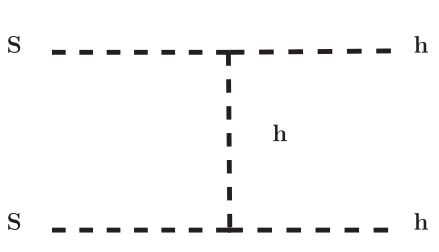

(d)

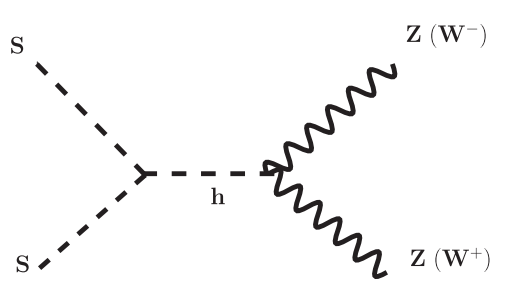

(e)

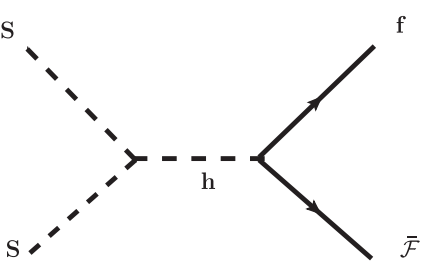

(f)

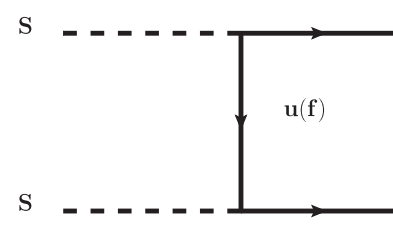

(g)

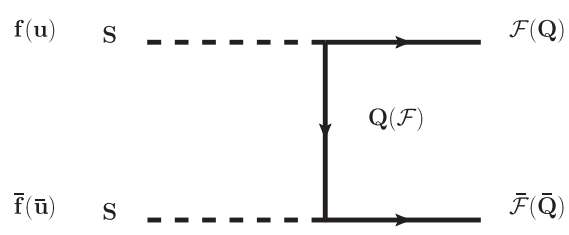

(h)

FIG. 1. Annihilation channels for scalar singlet dark matter $S$.

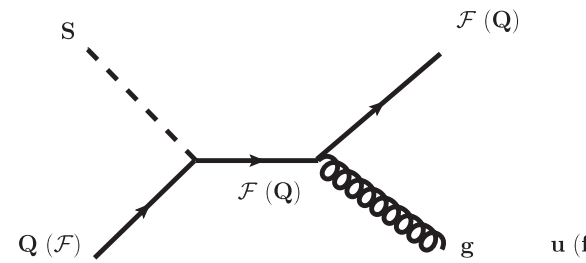

(a)

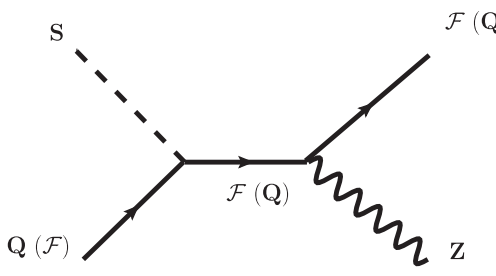

(d)

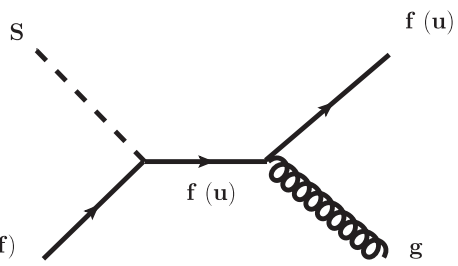

(b)

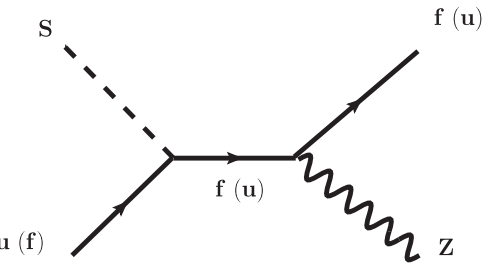

(e)

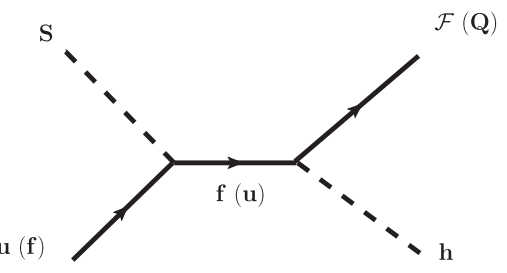

(g)

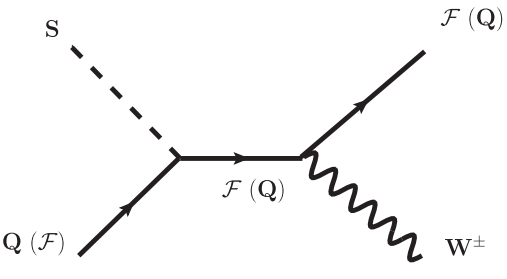

(c)

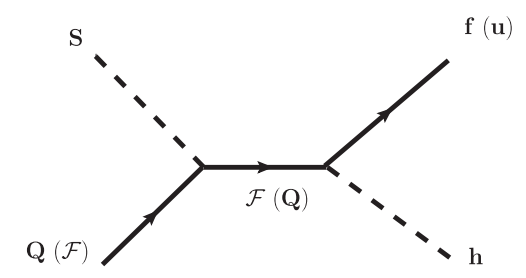

(f)

FIG. 2. Coannihilation channels for scalar singlet dark matter $S$. 


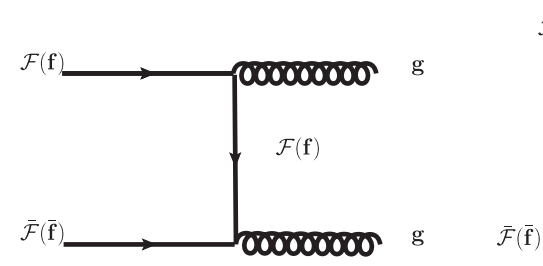

(a)

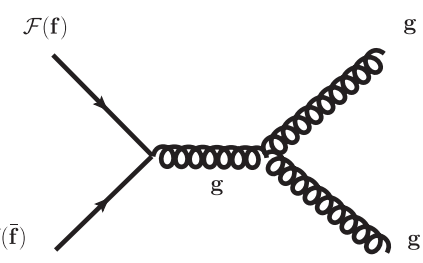

(b)

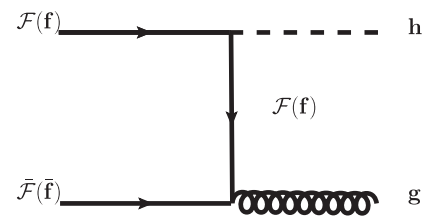

(c)

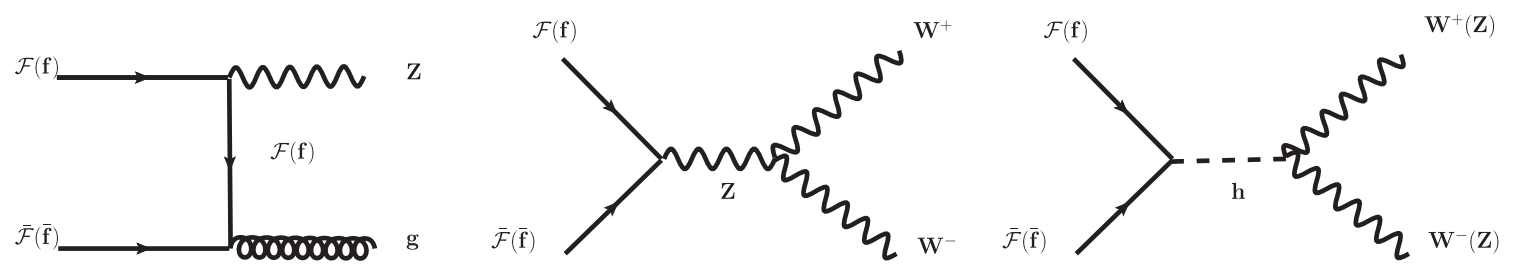

(d)

(e)

(f)

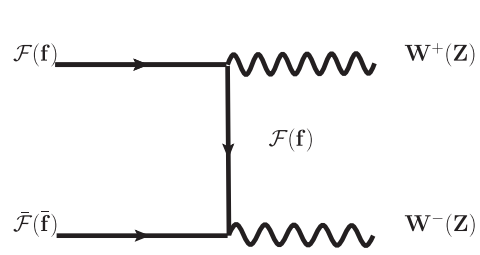

(g)

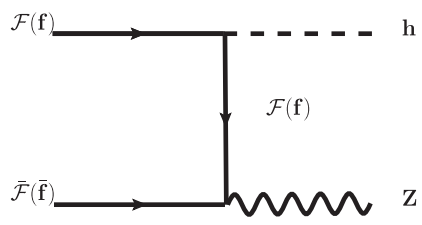

(h)

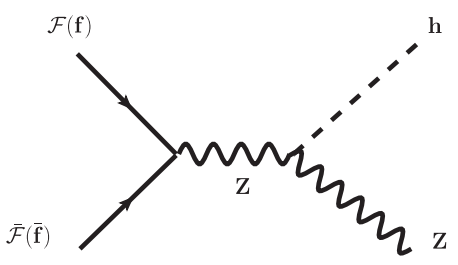

(i)

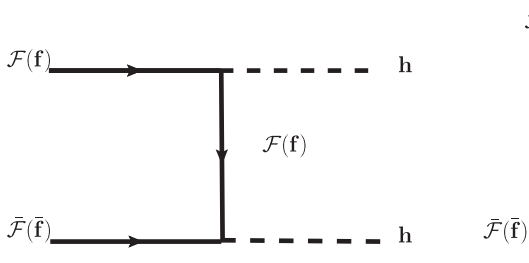

(j)

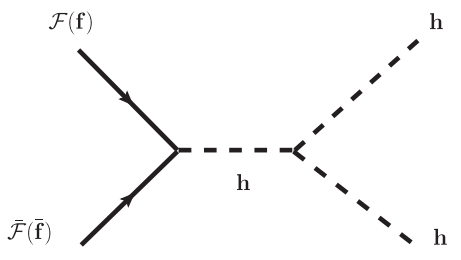

(k)

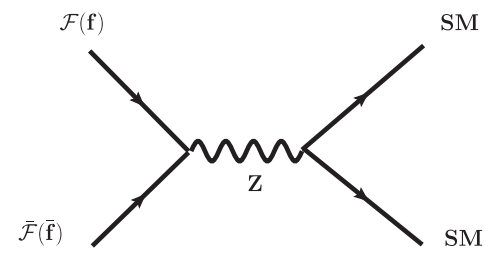

(1)

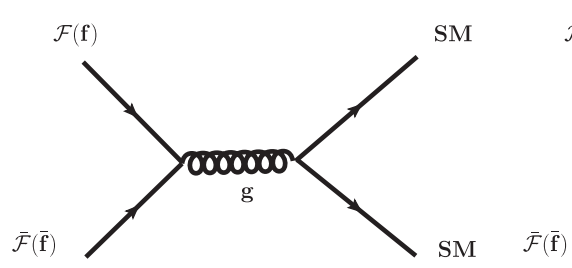

(m)

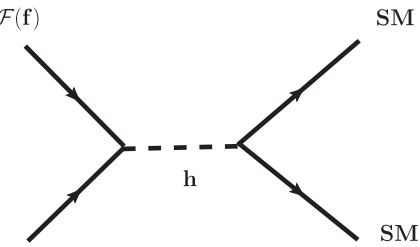

(n)

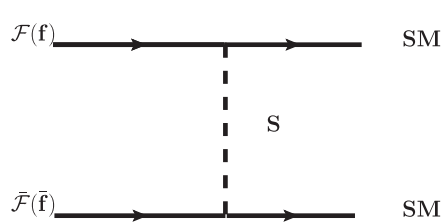

(o)

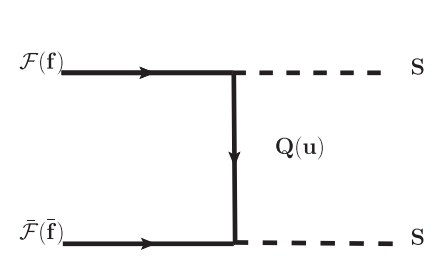

(p)

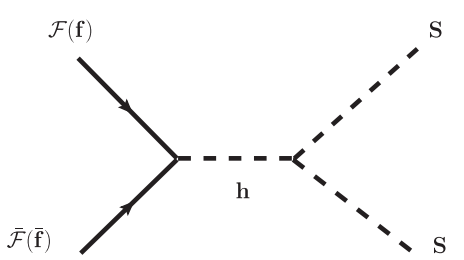

(q)

FIG. 3. Annihilation channels for vectorlike quarks. 


$$
\begin{aligned}
\langle\sigma|v|\rangle_{\mathrm{eff}}= & \frac{g_{s}^{2}}{g_{\mathrm{eff}}^{2}} \sigma(\bar{S} S)+2 \frac{g_{s} g_{\psi}}{g_{\mathrm{eff}}^{2}} \sigma\left(\bar{S} \psi_{i}\right)\left(1+\Delta_{i}\right)^{3 / 2} \exp \left[-x \Delta_{i}\right] \\
& +2 \frac{g_{\psi}^{2}}{g_{\mathrm{eff}}^{2}} \sigma\left(\bar{\psi}_{i} \psi_{j}\right)\left(1+\Delta_{i}\right)^{3 / 2}\left(1+\Delta_{j}\right)^{3 / 2} \\
& \times \exp \left[-x\left(\Delta_{i}+\Delta_{j}\right)\right] \\
& +\frac{g_{\psi}^{2}}{g_{\mathrm{eff}}^{2}} \sigma\left(\bar{\psi}_{i} \psi_{i}\right)\left(1+\Delta_{i}\right)^{3} \exp \left[-2 x \Delta_{i}\right] .
\end{aligned}
$$

In the equation above, $g_{s}$ and $g_{\psi}$ are the spin degrees of freedom for $S$ and $\psi_{i, j}$, where $\psi_{i, j}$ represents all the VLQs $\left(\mathcal{F}_{1}, \mathcal{F}_{2}, f\right)$ involved. Here, $x=\frac{m_{S}}{T}$ and $\Delta_{i}$ depict the mass splitting ratio $\frac{m_{i}-m_{S}}{m_{S}}$, where $m_{i}$ stands for the masses of all the VLQs. $g_{\text {eff }}$ in Eq. (18) is the effective degrees of freedom given by

$$
g_{\mathrm{eff}}=g_{s}+g_{\psi}\left(1+\Delta_{i}\right)^{3 / 2} \exp \left[-x \Delta_{i}\right] .
$$

Note that as the VLQs share the same $Z_{2}$ charge similar to the DM, their annihilations would also be important for evaluating the effective annihilation cross section. In the following analysis, we use the MICROMEGAS package [44] to find the region of parameter space that corresponds to correct relic abundance for our DM candidate satisfying PLANCK constraints [2],

$$
0.119 \lesssim \Omega_{\mathrm{DM}} h^{2}<0.121
$$

As mentioned earlier, the DM parameter space can be constrained significantly by the null result at different direct detection experiments such as LUX [8], PandaX-II [9,10] and XENON1T [11,12]. Apart from the usual SM Higgs mediated Feynman diagrams for the direct detection of the singlet scalar dark matter, the present setup has additional diagrams which come into the picture due to the presence of the VLQs. In Fig. 4 we show all the scattering processes of the DM $S$ with the detector nucleon.

\section{B. Results}

Based upon the above discussion, it turns out that the following set of parameters are the relevant ones for DM phenomenology:

$$
\left\{m_{S}, m_{1,2}, \sin \theta, \lambda_{H S}, \alpha_{1,2}\right\},
$$

whereas other parameters of the model, e.g., $y$ and bare masses, are derivable parameters from this set itself using Eq. (10). For simplicity, we set $\alpha_{1}=\alpha_{2}=\alpha$ unless otherwise mentioned. Since we specifically look for reopening the window of scalar singlet DM (which is otherwise ruled out) in the intermediate mass range $\sim(200-950) \mathrm{GeV}$ through the coannihilation process with the VLQs, we expect these VLQs to be heavier but having mass close to DM mass. However from the point of view of the LHC accessibility in future, it would be interesting to keep their masses lighter than $1 \mathrm{TeV}$. With this, the value of $\sin \theta$ is expected to be small as seen from Eq. (9), unless there exists a very fine-tuned mass difference $\Delta_{21}$ between the bare masses $\left(M_{\mathcal{F}}\right.$ and $\left.M_{f}\right)$ of VLQs. As a benchmark value, we consider $\Delta_{21}=50 \mathrm{GeV}$. Note that with such a choice, the Yukawa coupling $y$, obtainable via relation Eq. (10c) for fixed choices of $\Delta_{21}$ and $\sin \theta$, can be kept well below 1 provided $\sin \theta$ is small. We have taken here a conservative choice with $\sin \theta=0.1$. Note that a small value of $y$ (significantly below 1) turns out to be quite natural from the point of view of maintaining perturbativity of $y$. It is also found in [39] that $y$ above 0.3 can make the Higgs quartic coupling $\lambda_{H}$ negative at some high scale and thereby could be dangerous for vacuum stability.

To facilitate our discussion on DM parameter space of the model under consideration, we first provide the contour plot for correct relic density consistent with Planck data in the $\lambda_{H S}-m_{S}$ plane as shown in the left panel of Fig. 5. We also impose the DM-nucleon direct search cross section limit as obtained from the XENON1T experiment [12]. The relic as well as DD cross section satisfied points are indicated by the orange portion while points in the purple portion of the contour indicate the relic-satisfied but otherwise excluded by DD limits. The change in the DD cross section versus $m_{S}$ variation with different choices of Higgs portal couplings is represented in the right panel. It turns out that singlet scalar DM mass below $1 \mathrm{TeV}$ is essentially ruled out except in the SM Higgs resonance region. This finding is also consistent with the results of other works $[45,46]$.

In Fig. 6 (left panel), we have shown the variation of relic density $\Omega_{S} h^{2}$ against the Higgs quartic coupling $\lambda_{H S}$ in our

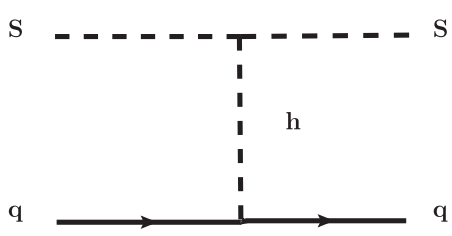

(a)

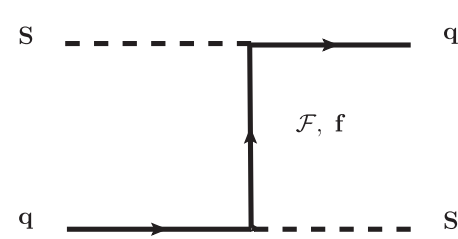

(b)

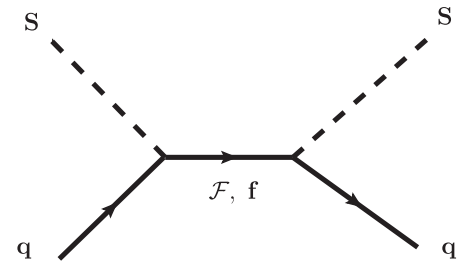

(c)

FIG. 4. Spin independent elastic scattering of the DM nucleon. 


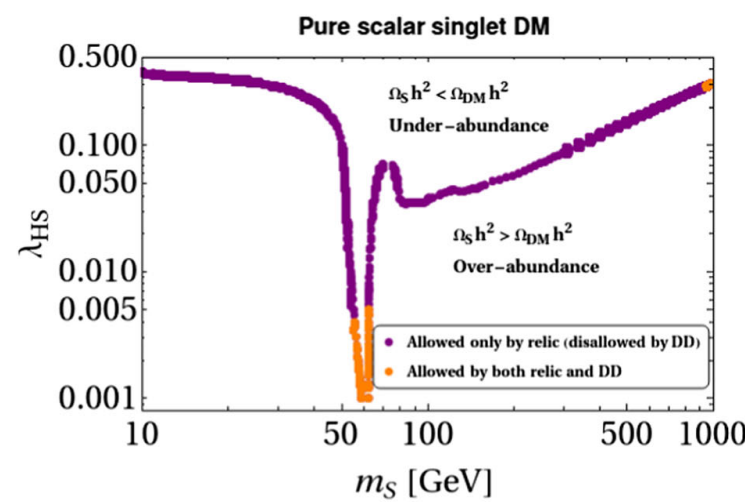

(a)

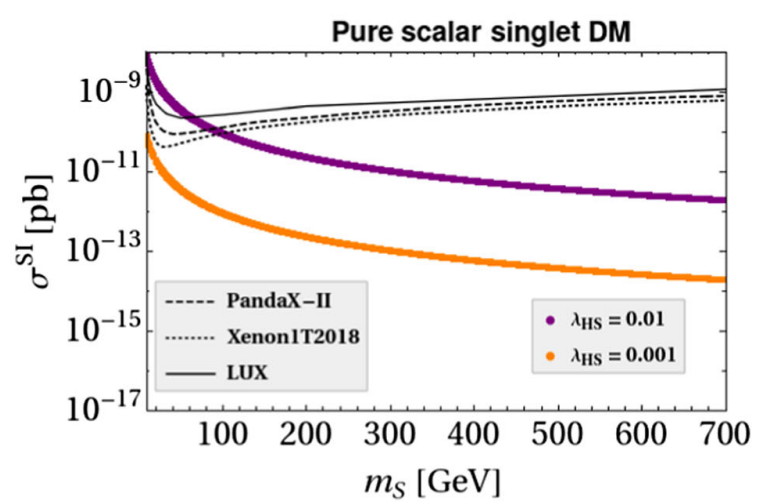

(b)

FIG. 5. Relic density and direct search/XENON1T allowed parameter space of the scalar singlet $(S)$ DM on the left (in the $\lambda_{H S}-m_{S}$ plane). On the right we show the spin independent DM-nucleon cross section for a scalar singlet DM for two values of $\lambda_{H S}=0.01,0.001$.

setup, while mass difference between the two up-type VLQs $\left(\Delta_{21}\right)$, DM mass and mixing angle $\sin \theta$ are kept fixed at $50 \mathrm{GeV}, 500 \mathrm{GeV}$ and 0.1 respectively. Such a choice of DM mass is guided by our aim to explore the otherwise disallowed range of DM mass for scalar singlet $\mathrm{DM}$, as stated before. The respective plots corresponding to different $m_{1}$ masses are indicated by green $(555 \mathrm{GeV})$, red $(565 \mathrm{GeV})$ and blue $(575 \mathrm{GeV})$ lines. The value of the other remaining parameter $\alpha$ is varied in a range, $0.001 \leq$ $\alpha \leq 0.1$, while generating the plots.

We find (refer to Fig. 6, left panel) that the relic can be satisfied by a relatively (compared to the pure singlet scalar case) small value of $\lambda_{H S} \lesssim 0.1$, thanks to the effect of coannihilations (inclusive of diagrams in Figs. 2 and 3) involving the interactions provided in Eq. (1) and the gauge interactions. The presence of this coannihilation is more transparent when we observe that with the increase of $m_{1}$, the relic becomes larger. This is simply because with increased values of $m_{1}, \Delta_{1,2}$ become larger and hence the effect of coannihilation reduces [see Eq. (18)] which contributes to a smaller effective annihilation cross section and hence larger relic is obtained. The small $\lambda_{H S}$ turns out to be helpful in evading the DD limits. Since some of the couplings involved in coannihilation processes, e.g., couplings $\alpha$, are also involved in the DD process, their effects become crucial in getting the correct DM relic density as well as to satisfy DD bounds. It can be noticed that for $\lambda_{H S}$ below 0.1 , the effect of coannihilation (actually the annihilations of VLQs) remains so important that the relic is essentially insensitive to the change of $\lambda_{H S}$. However this conclusion changes when $\lambda_{H S}$ crosses 0.1 . There we notice a significant fall in the relic plots (with different $m_{1}$ value) as well as a merger of them. This is because in this regime, annihilation of DM provides a significant contribution to

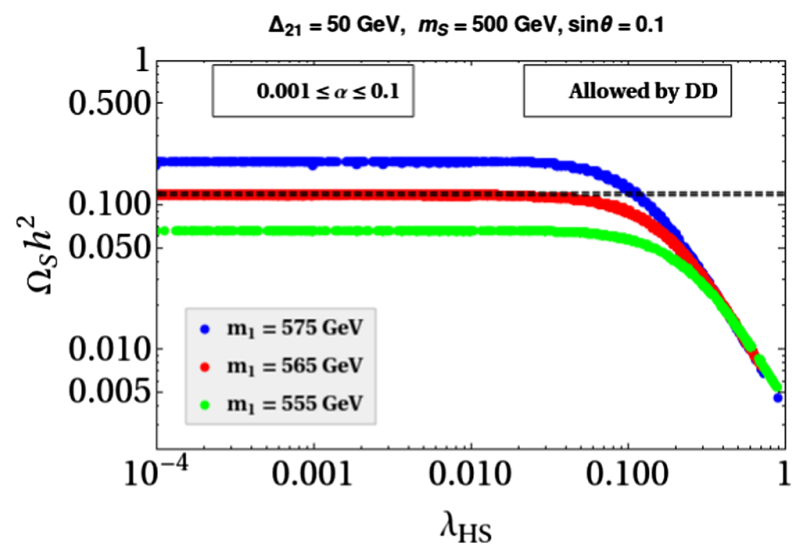

(a)

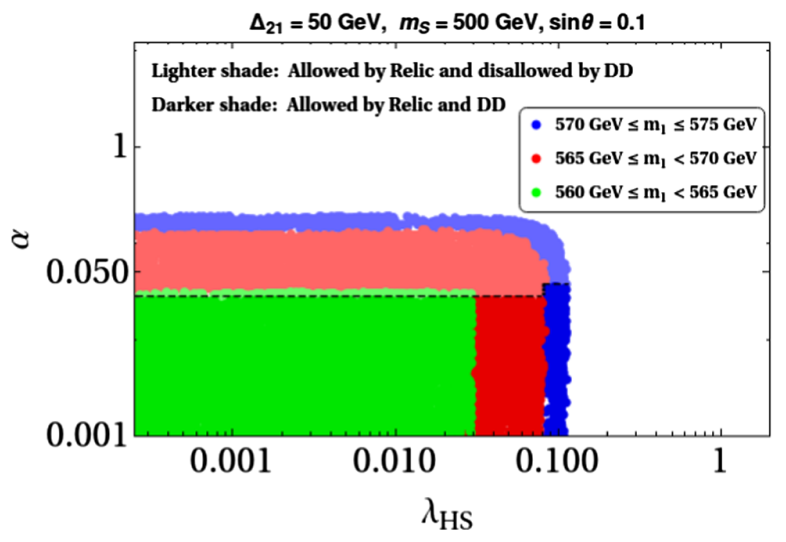

(b)

FIG. 6. The left panel shows the variation of $\Omega_{S} h^{2}$ with $\lambda_{H S}$ for different values of lightest VLQ mass $\left(m_{1}\right)$ while the right one describes the allowed (disallowed) region for $\alpha-\lambda_{H S}$ parameters denoted by a darker (lighter) shade. The black dotted line (horizontal) in the left panel corresponds to the correct relic density. The black dotted line in the right panel segregates the allowed region (below this line, having dark shaded points) from the disallowed region (above this line, having light shaded points) by direct detection results. 


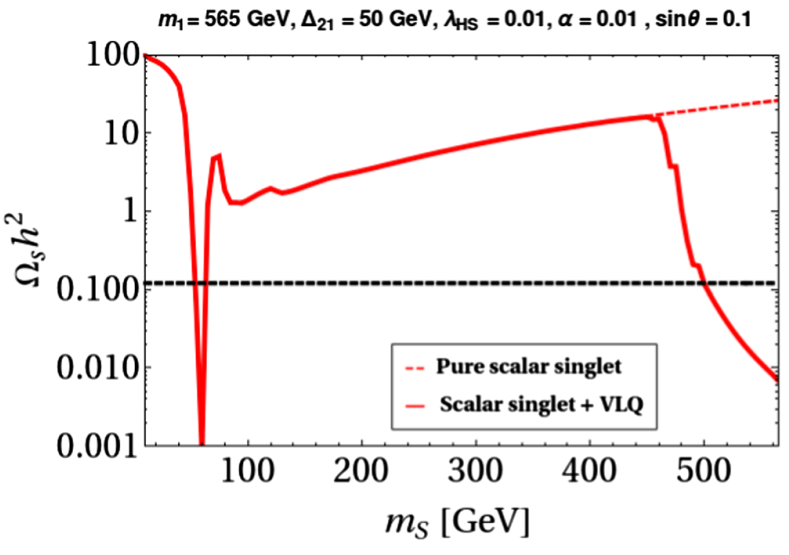

FIG. 7. Relic density plot comparing the scenario for scalar singlet DM with that of scalar singlet DM in the presence of vectorlike quarks with $\lambda_{H S}=0.01$.

effective annihilation cross section $\langle\sigma v\rangle_{\text {eff }}$ and coannihilation, although present, becomes less important. So overall the decrease in relic beyond $\lambda_{H S} \gtrsim 0.1$ is effectively related to the increase in $\lambda_{H S}$ in the usual fashion.

In the right panel of Fig. 6 we show the parameter space in the $\alpha-\lambda_{H S}$ plane, where the points with lighter shades are allowed only by the relic density and disallowed by the direct search experiments, whereas the darker shaded region (below the black dotted line separating the allowed and disallowed regions) corresponds to the parameter space which is allowed by both the relic density as well as the direct search constraints. In generating the plot, all other parameters except $m_{1}$ are kept at the same values as in the plot of the left panel. $m_{1}$ here is varied in three ranges of values as indicated by the color code mentioned in the inset of the figure. As with the increase of the $m_{1}$ value (such that $\Delta_{1}$ also increases), the possibility of coannihilation becomes less, the respective relic and DD satisfied region also shrinks. One can also notice that higher values of $\alpha$,

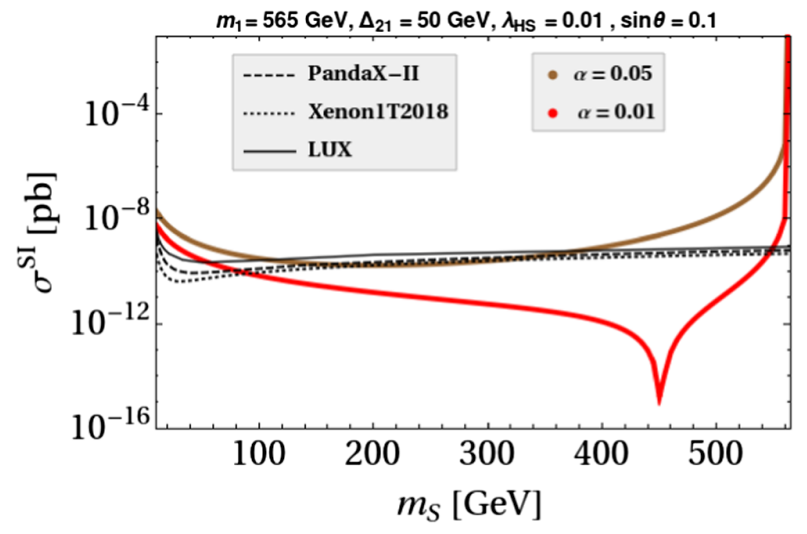

(a) beyond $\sim 0.05$ or so, are disfavored by the DD searches. The reason would be clear if we look at the Feynman diagrams in Figs. 2 and 4. A large $\alpha$ would lead to a cross section relatively large compared to the DD limits. Hence it turns out that in the regime of parameters with small values for both $\lambda_{H S}$ and $\alpha$, it is the VLQ annihilations via gauge interactions (as part of coannihilations via Fig. 3) which play a crucial role in getting the correct relic density.

A variation of relic $\Omega_{S} h^{2}$ with mass of the DM $m_{S}$ is shown in Fig. 7 indicated by a red line where $m_{1}, \lambda_{H S}, \Delta_{21}, \alpha$ and $\sin \theta$ are fixed at $565 \mathrm{GeV}, 0.01,50 \mathrm{GeV}, 0.01$ and 0.1 respectively as also mentioned on the top of the figure. While compared with the case of a pure singlet DM having Higgs portal coupling set at 0.01 (the dashed red line), we notice several interesting features. First, the pattern is very much similar until $m_{S}$ approaches $450 \mathrm{GeV}$ or so. Beyond this point, the coannihilations start to be dominant as the $\Delta_{1}$ becomes small as seen from Eq. (18). Hence due to the sudden increase in the effective-annihilation cross section, the relic abundance falls rapidly. With the $m_{1}$ value fixed at $565 \mathrm{GeV}, m_{S}$ cannot exceed $m_{1}$.

In Fig. 8 (left panel), we provide the DD cross section versus $m_{S}$ in our setup for two different choices of $\alpha$. While $\alpha=0.01$ is allowed for most of the intermediate mass range of DM, $\alpha=0.05$ remains above the DD limits set by XENON1T data, in accordance with the contribution followed from Fig. 6. Also there is a noticeable dip in the plot which is indicative of the relative sign difference between these $s$ and $t$ channel contributions. In the right panel, we explore what happens if we deviate from our simplified assumption of keeping both $\alpha_{1}$ and $\alpha_{2}$ the same. We find that in this case $\alpha_{1}=0.01$ and $\alpha_{2}=0.03$ are also an allowed set of choices. However in terms of usefulness of VLQs in bringing back the otherwise disallowed intermediate mass range of a singlet scalar DM, our conclusion remains unaltered. In the following section we extend our discussion for EW vacuum stability and,

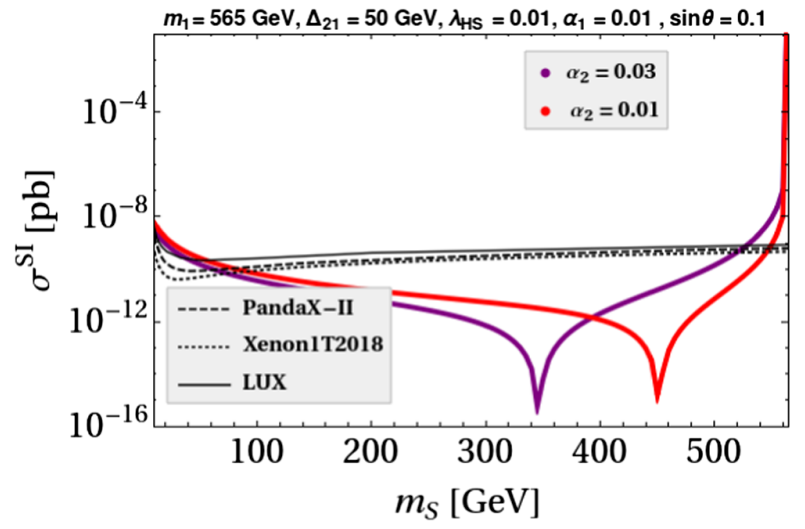

(b)

FIG. 8. Spin independent direct detection cross section for: (a) two different values of $\alpha=0.05,0.01$ and (b) fixed value of $\alpha_{1}=0.01$ and two different values of $\alpha_{2}=0.03,0.01$. 
in the next, we find the parameter space consistent with both the DM phenomenology as well as vacuum stability.

\section{ELECTROWEAK VACUUM STABILITY}

In this section, we aim to discuss the impact of the inclusion of the singlet scalar and VLQs on EW vacuum stability in our setup. It is well known that the absolute stability of the SM Higgs vacuum can be ensured by $\lambda_{H}(\mu)>0$ at any energy scale $\mu$ provided the EW minimum is the global one. In the presence of an additional deeper minimum other than the $\mathrm{EW}$ one, one has to evaluate the tunneling probability $P_{T}$ of the EW vacuum to this second minimum given by $P_{T}=T_{U}^{4} \mu_{B}^{4} e^{-8 \pi^{2} /\left(3\left|\lambda_{H}\left(\mu_{B}\right)\right|\right)}$. Here $T_{U}$ is the age of the Universe, $\mu_{B}$ is the scale at which probability is maximized, to be obtained from $\beta_{\lambda_{H}}\left(\mu_{B}\right)=0$ where $\beta_{\lambda_{H}}$ is the beta function for SM Higgs quartic coupling. The Universe would then be a metastable one if the decay lifetime of the EW vacuum to the second one is longer than the age of the Universe, $T_{U}$. This metastability requires

$$
\lambda_{H}\left(\mu_{B}\right)>\frac{-0.065}{1-0.01 \ln \left(\frac{v}{\mu_{B}}\right)} .
$$

Within the SM alone, it is the top quark Yukawa coupling, $y_{t}$, which drives the SM Higgs quartic coupling to a negative value at around $10^{10} \mathrm{GeV}[17,18,20,22,47]$. However within the present limits on the top quark mass, the EW vacuum turns out to be a metastable one. In our setup, inclusion of singlet scalar $S$ as DM and the VLQs would affect this situation. The presence of an additional singlet scalar is known to generate a positive contribution to the beta function of $\lambda_{H}$ through the interaction like $\frac{\lambda_{H S}}{2} H^{\dagger} H S^{2}$ and thereby helps in driving the Higgs vacuum toward more stability $[38,48-55]$. It is found that, in order to make the Higgs vacuum absolutely stable up to $M_{\mathrm{Pl}}$, the scalar singlet (here DM) mass should fall above $\sim \mathrm{TeV}$. As in this paper, we focus on the intermediate range of DM mass, its sole presence cannot be effective in achieving the absolute vacuum stability. On the other hand, we also have the VLQs. It turns out, as discussed below, their presence would be helpful in achieving the absolute stability of the EW vacuum even with the scalar singlet DM mass below $1 \mathrm{TeV}$.

We have already seen the important role of these VLQs in the present setup in making the otherwise disallowed parameter space (say in terms of mass) a viable one by providing additional contributions to DM effective annihilation cross section and in DD diagrams. Following the results of [39], these VLQs also help in achieving the electroweak vacuum stable up to a large scale. This can be understood if we look into their positive (additional) contribution to the beta function of the gauge couplings $g_{1,2,3}$ associated with $S U(3), S U(2)$ and $U(1)$ gauge group of the SM respectively (at one loop) given by $\beta g_{1}=\beta_{g_{1}}^{\mathrm{SM}}+\beta_{g_{1}}^{\mathrm{VLQ}}=\beta_{g_{1}}^{\mathrm{SM}}+\frac{g_{1}^{3}}{16 \pi^{2}}\left[\frac{4}{5} N_{c}\left(2 n_{2} Y_{\mathcal{F}}^{2}+n_{1} Y_{f^{\prime}}^{2}\right)\right]$

$$
\begin{gathered}
\beta g_{2}=\beta_{g_{2}}^{\mathrm{SM}}+\beta_{g_{2}}^{\mathrm{VLQ}}=\beta_{g_{2}}^{\mathrm{SM}}+\frac{g_{2}^{3}}{16 \pi^{2}}\left(\frac{2}{3} N_{c} n_{2}\right) \\
\beta g_{3}=\beta_{g_{3}}^{\mathrm{SM}}+\beta_{g_{3}}^{\mathrm{VLQ}}=\beta_{g_{3}}^{\mathrm{SM}}+\frac{g_{3}^{3}}{16 \pi^{2}}\left(\frac{2 n_{3}}{3}\right),
\end{gathered}
$$

where color charge $N_{c}=3$ for any fermion (SM or VLQ) belonging to the fundamental representation of $S U(3), n_{1}$ and $n_{2}$ represent number of $S U(2)$ singlet and doublet VLQs respectively while $n_{3}$ corresponds to the number of $S U(3)$ triplet vectorlike fields. $Y_{f^{\prime}}$ and $Y_{\mathcal{F}}$ are the hypercharges of the VLQ singlets and doublets respectively. The VLQs being charged under $S U(3)$ increase the number of colored particles in the present setup and hence effectively increases the $\beta$ function of the gauge couplings, noticeably for the $g_{3}$. As a result of this, the running of the top Yukawa coupling $y_{t}$ experiences a sizable decrease in its value (compared to its value within SM alone) at high scale predominantly due to the involvement of the $-8 y_{t} g_{3}^{2}$ term in $\beta_{y_{t}}^{\mathrm{SM}}$ as

$$
\begin{aligned}
\beta y_{t}= & \beta_{y_{t}}^{\mathrm{SM}}+\beta_{y_{t}}^{\mathrm{VLQ}}=\frac{y_{t}}{16 \pi^{2}}\left[\frac{9}{2} y_{t}^{2}+2 N_{c} y^{2}-\frac{17}{20} g_{1}^{2}\right. \\
& \left.-\frac{9}{4} g_{2}^{2}-8 g_{3}^{2}+2 N_{c} y^{2}\right] .
\end{aligned}
$$

Provided this decrease in $y_{t}$ is significant enough, it may no longer drag the $\lambda_{H}$ towards the negative value [recall that it was the $-6 y_{t}^{4}$ term present in $\beta_{\lambda_{H}}$ which pushes $\lambda_{H}$ to negative in $\mathrm{SM}$ at around $10^{9-10} \mathrm{GeV}\left(\equiv \Lambda_{I}^{\mathrm{SM}}\right)$ depending on the top quark mass], rather keeps it positive all the way until the Planck scale.

One should also note that due to the involvement of both doublet as well as singlet VLQs in our framework, Yukawa interaction involving the SM Higgs and these VLQs will also come into the picture [see Eq. (1)]. The associated coupling $y$ involved in this interaction may result in negative contribution in the running of $\lambda_{H}$ as seen from

TABLE II. Values of the relevant SM couplings [top-quark Yukawa $y_{t}$, gauge couplings $g_{i}(i=1,2,3)$ and Higgs quartic coupling $\lambda_{H}$ ] at energy scale $\mu=m_{t}=173.2 \mathrm{GeV}$ with $m_{h}=$ $125.09 \mathrm{GeV}$ and $\alpha_{S}\left(m_{Z}\right)=0.1184$.

\begin{tabular}{lccccc}
\hline \hline Scale & $\lambda_{H}$ & $y_{t}$ & $g_{1}$ & $g_{2}$ & $g_{3}$ \\
\hline$\mu=m_{t}$ & 0.125932 & 0.93610 & 0.357606 & 0.648216 & 1.16655 \\
\hline \hline
\end{tabular}


TABLE III. Benchmark point which satisfies the correct relic density and is allowed by the direct detection experiments for $\sin \theta=0.1$.

\begin{tabular}{lccccccc}
\hline \hline BP & $m_{S}[\mathrm{GeV}]$ & $m_{1}[\mathrm{GeV}]$ & $\Delta_{21}[\mathrm{GeV}]$ & $\lambda_{H S}$ & $\alpha$ & $\Omega_{S} h^{2}$ & $\sigma_{S}^{S I}(p b)$ \\
\hline BP I & 500 & 565 & 50 & 0.01 & 0.01 & 0.119 & $7.169 \times 10^{-12}$ \\
\hline \hline
\end{tabular}

$$
\begin{aligned}
\beta \lambda_{H} & =\beta_{\lambda_{H}}^{\mathrm{SM}}+\beta_{\lambda_{H}}^{\mathrm{VLQ}}+\beta_{\lambda_{H}}^{S(1)}, \\
& =\beta_{\lambda_{H}}^{\mathrm{SM}}+\frac{2 n_{F}}{16 \pi^{2}}\left(4 N_{c} y \lambda_{H}-2 N_{c} y^{4}\right)+\frac{1}{2} \lambda_{H S}^{2},
\end{aligned}
$$

where $n_{F}$ is the number of families of VLQ multiplets (both doublet and singlet) participating in the Yukawa interaction with SM Higgs. In fact, it has been shown in [39] that $y>\mathcal{O}(0.3)$ can make $\lambda_{H}$ negative at the large scale. However in our analysis we have found that this coupling does not have a significant impact on the DM phenomenology and hence we can keep it rather small throughout. Note that this smallness is in fact supported by the small mixing angle $\theta$ and $\Delta_{21} \sim \mathcal{O}(100) \mathrm{GeV}$ as can be seen using Eq. (10).

For the analysis purpose in both the VLQ as well as the VLL scenarios, we run the two-loops RG equations for all the SM couplings as well as all the other relevant BSM coupling involved in both the setups from $\mu=m_{t}$ to $M_{\mathrm{Pl}}$ energy scale. We use the initial boundary values of all SM couplings as given in Table II at an energy scale $\mu=m_{t}$. The boundary values have been evaluated in [20] by taking various threshold corrections at $m_{t}$ and the mismatch between top pole mass and $\overline{\mathrm{MS}}$ renormalized couplings into account. Here, we consider $m_{h}=125.09 \mathrm{GeV}$, $m_{t}=173.2 \mathrm{GeV}$, and $\alpha_{S}\left(m_{Z}\right)=0.1184$. We only provide the one-loop $\beta$ functions for both of the scenarios namely, VLQ and VLL in Appendices A and B respectively. The $\beta$ functions were generated using the model implementation in SARAH [56].

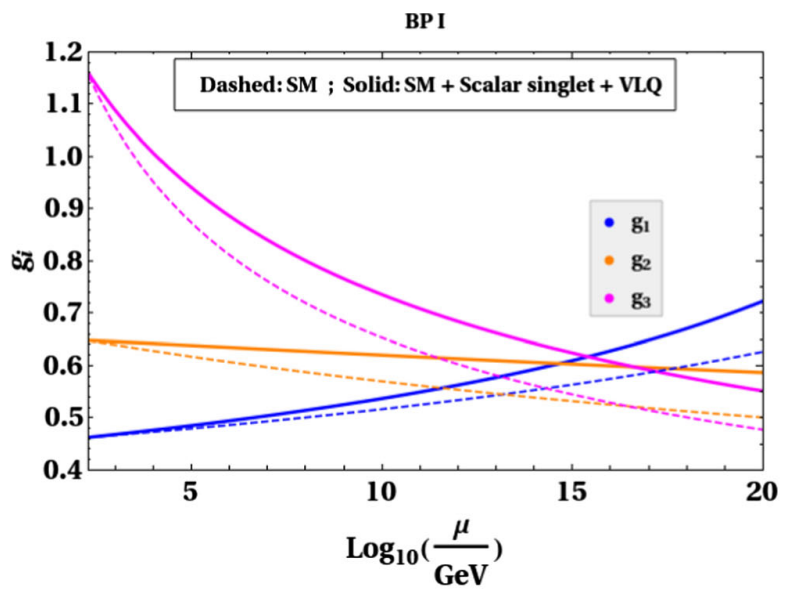

(a)
In continuity with the discussion above, we now provide plots for the running of the gauge couplings, Yukawa coupling $y_{t}$ and Higgs quartic coupling $\lambda_{H}$. For this purpose, we consider the set of benchmark values of parameters of our setup denoted by BP I in Table III, the choice of which are mainly motivated from the DM phenomenology discussed before.

In the last two columns, we specify the respective contribution to the relic and DD cross section estimate followed from this particular choice of parameters, BP I. Using this, we first show the effect of VLQs on gauge couplings $g_{1}$ (in blue), $g_{2}$ (in orange) and $g_{3}$ (in purple) in the left panel of Fig. 9. While their running in SM is represented in dotted lines, the respective running of them in the case of the present model (SM + VLQs + scalar singlet) is denoted by the solid lines.

While the positive shift to $g_{3}$ is expected due to the inclusion of VLQs, the rise in $g_{1}, g_{2}$ values is due to the presence of the extra $S U(2)$ doublet, i.e., $\mathcal{F}$ in our framework. In Fig. 9(b) we compare the running of Higgs quartic coupling in our model (solid line) with that of the SM (dashed line) and scalar singlet extension of the SM (dotted line). Note that with the choice of $\lambda_{H S}=0.01$, introduction of the scalar singlet does not make any significant contribution on the SM prediction of the $\beta$ function. As we have discussed above, the presence of VLQs alters the running of the gauge couplings with a positive shift and hence top quark Yukawa, $y_{t}$, has a prominent drop at high scale. In Fig. 10, this downward shift in the running of the top Yukawa coupling (due to the negative contribution coming from the gauge coupling $g_{3}$ and others) is shown.

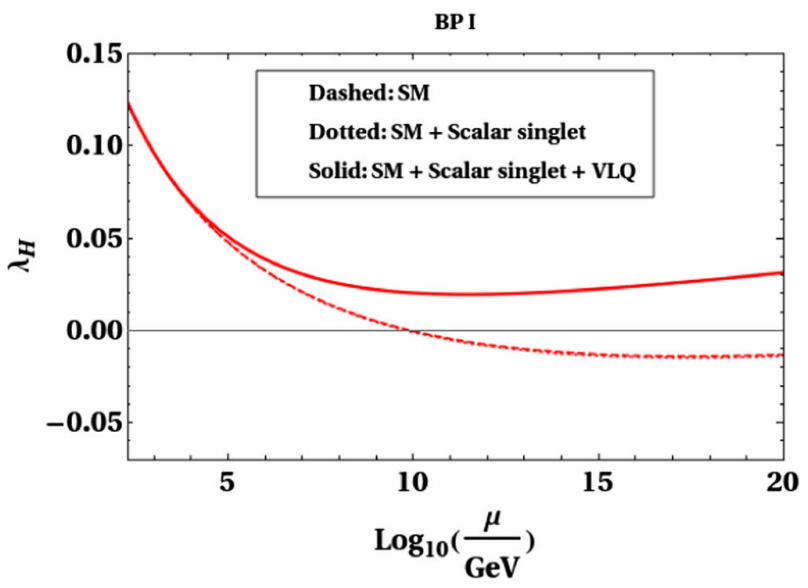

(b)

FIG. 9. Running of (a) gauge couplings and (b) Higgs quartic coupling. 


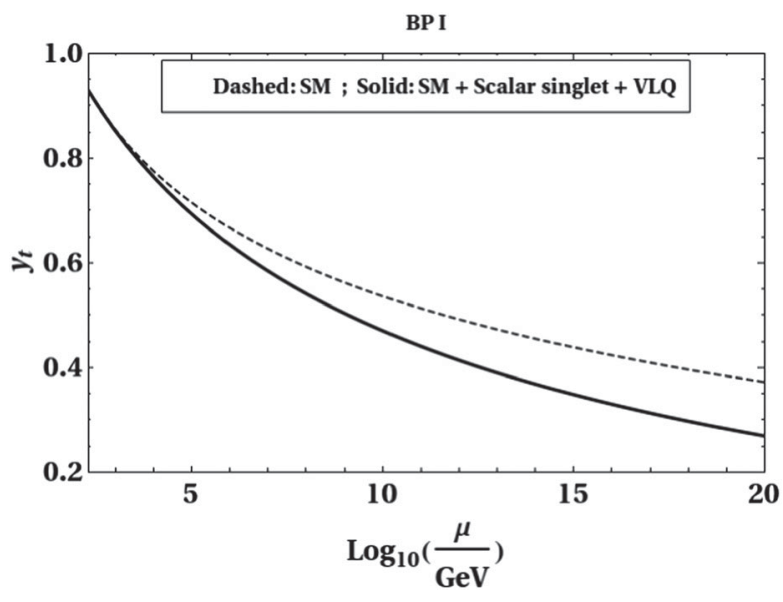

FIG. 10. Running of top Yukawa coupling.

\section{COMBINED ANALYSIS OF DM AND EW VACUUM STABILITY}

Figure 11 shows the allowed parameter space in the $m_{1}-m_{S}$ plane where the dark matter relic and DD constraints are imposed. In obtaining this plot, parameters other than $m_{1}, m_{S}$ and $\alpha$ are kept fixed at their respective benchmark values (BP I of Table III). Note that the patch with red dots associated to $\alpha=0.01$ spans over the entire mass range of dark matter $m_{S}: 250 \mathrm{GeV}$ to $1 \mathrm{TeV}$ while a slight increase in $\alpha(=0.02)$ restricts $m_{S}$ to be above $\sim 450 \mathrm{GeV}$. A similar trend follows for larger values of $\alpha=0.03(0.04)$ for which the allowed DM mass starts from $650 \mathrm{GeV}(925 \mathrm{GeV})$. This happens due to the combined impact of (i) the involvement of the mass splitting $m_{1}-m_{S}$ in determining the relic density and (ii) sensitivity of the dark matter DD to $\alpha$. The correlation between $m_{1}$ and $m_{S}$ as seen in Fig. 11 follows mainly because the relic density requirement coannihilations of VLQs to SM particles are the relevant processes that bring the relic density of scalar singlet DM at an appropriate level. As we have discussed before, this depends crucially on the mass splitting of $m_{1}-m_{S}$. However as it turned out from our understanding of

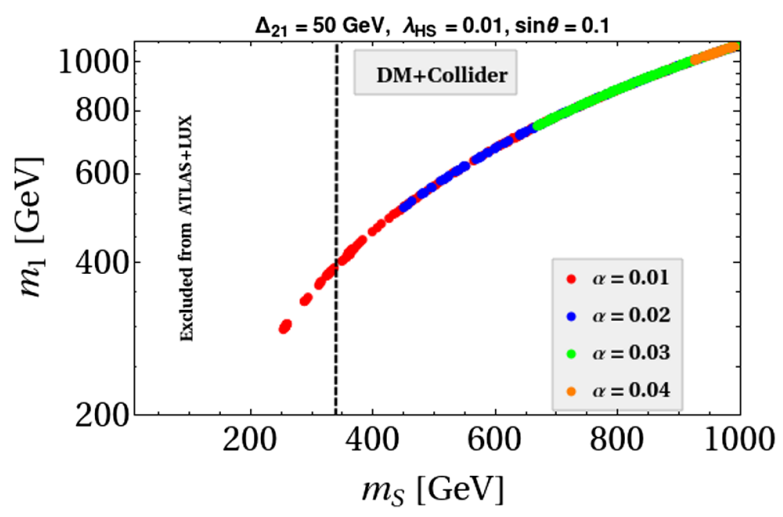

FIG. 11. Allowed parameter space in the $m_{1}-m_{S}$ plane.
DM phenomenology, relic density is almost insensitive to $\alpha$ though the DD cross section depends on it. Hence an increase in $\alpha$ value would also enhance the DD cross section (thereby excluded) unless the mass of the DM is also increased (as cross section varies inversely with $m_{s}$ ). This explains why with the increase of $\alpha$, the DM mass should also be increased (as seen going from red $\rightarrow$ blue $\rightarrow$ green and then to orange patches in the plot).

Now turning back to the vacuum stability issue, we note that presence of VLQs allows the EW minimum to be absolutely stable all the way up to $M_{\mathrm{Pl}}$. However this conclusion is independent to the choice of $\alpha$ or, in other words, $\alpha$ remains unrestricted. In our setup, we have Yukawa interaction of the VLQs to the DM, guided by $\alpha$. Now with the inclusion of DM phenomenology, we restrict the coupling $\alpha$. In conclusion, we have ended up in a situation, where a scalar singlet DM having intermediate mass range (in particular 250-900 GeV) now becomes allowed in the presence of VLQs and simultaneously can make the EW stable.

In this scenario with VLQs, as we have seen, there arises several new annihilation and coannihilation channels due to the additional interactions of DM present in Eq. (1). These are shown in terms of Feynman diagrams in Figs. 1 and 2. Due to the presence of the last two terms in the Eq. (1), one can see that the dark matter $S$ can also annihilate into gluon pair $(g g)$ at one loop (through box diagram). It has been shown in [33-37] that with a larger choice of Yukawa coupling (here $\alpha$ ) this annihilation of $S$ to the gluon pair can provide a significant contribution to the relic of $S$. In [34], the authors notice that for the mass regime $m_{S}<m_{t}$ with the Yukawa coupling of $\sim \mathcal{O}(10)$ the correct relic density can be satisfied. This large Yukawa coupling (involving upquark) however can create a problem in the DD of the dark matter as it will generate a large spin independent cross section and hence can be on the verge of being excluded by the DD searches. One should note that the amplitude of $S S \rightarrow g g$ depends on the square of the product of this Yukawa coupling and the strong gauge coupling. Hence this process will dominate over the other annihilation or the coannihilation channels of $S$ only if the associated Yukawa coupling is large. In our scenario, first of all, we focus on the scalar DM having mass above the top threshold. Second, we have used a small Yukawa in general, i.e., $\alpha \sim 0.01$, which helps in evading the DD constraint. Hence $S S \rightarrow g g$ (and other processes like $s s \rightarrow \gamma \gamma$, three body final states etc.) remains suppressed in our case.

We find that the annihilations of VLQs take part significantly in the effective DM annihilation cross section in this scenario. As shown in earlier works $[33,36,57,58]$, such annihilations of the two VLQ particles, in the nonrelativistic limit, can be affected by the nonperturbative Sommerfeld corrections through gluonic exchanges. It has been noticed that this correction may affect the relic abundance calculation by at most $15 \%$ (see $[33,36]$ for a recent discussion and references therein). In the present setup we have not included 
this correction, we leave it for a more complete exploration as a future study. One should also take into account the possible formation of the bound states due to the presence of the VLQs. It has however been concluded $[59,60]$, using the setup similar to ours except that only SM singlets are involved there, that the bound state has only moderate impact on the relic density and hence negligible.

It might be pertinent here to discuss the possible signatures of these additional colored particles in colliders. First, note that the lightest VLQ decaying into SM quark and $W$ boson leading to a prominent signature through jets $+\mathscr{E}_{T}+$ charged lepton [42] is forbidden in our setup as an artifact of $Z_{2}$ symmetry (for which the direct Yukawa interaction involving VLQs, Higgs and SM quarks is absent). Second, the possibility to trace back VLQ's signature through its decay into top quark and dark matter leading to $\mathbb{E}_{T}+$ hard jets also becomes nonexistent due to the unavailability of the phase space with the choice of parameters dictated by the DM phenomenology. The mass spitting between $m_{S}$ and $m_{1}$ turns out to be $\sim \mathcal{O}(50) \mathrm{GeV}$ as $\operatorname{seen}^{1}$ from Fig. 11. As we have discussed in Sec. III, the presence of these VLQs in the current setup can only be felt in the collider through their subsequent decay into the DM particle $S$ and light SM quark (or antiquark). Hence missing energy $\left(\mathscr{E}_{T}\right)$ plus multijets (arising due to gluon emission) would be the signal at LHC. Though the study in [33] shows that a VLQ mass $\sim 550-750 \mathrm{GeV}$ is allowed with $m_{S} \sim 500 \mathrm{GeV}$, such a constraint too will not be directly applicable here. This constraint came primarily due to the presence of large Yukawa coupling between VLQs, DM and the SM quark required to satisfy the DM relic density in their scenario. In our case, the correct relic is effectively produced by the annihilation of VLQs and the Yukawa coupling $\alpha$ is restricted to be small (to satisfy the DD bound). In fact, $\alpha$ is kept well below 0.1 in our case (see Fig. 11). Still in Fig. 11 we apply $m_{1}>400 \mathrm{GeV}$ and $m_{S}>$ $300 \mathrm{GeV}$ (discussed in [33]) as a conservative bound (indicated by the dotted vertical line). In the present work, we do not perform a detailed collider study to obtain precise bounds on mass of the VLQs. However it seems that the study would be rich due to the presence of both doublet and singlet VLQs and can surely be taken as a future endeavor.

\section{SCALAR SINGLET DM WITH VECTORLIKE LEPTONS}

In this section, we investigate a similar setup where vectorlike quarks are replaced with vectorlike leptons.

\footnotetext{
${ }^{1}$ The heaviest VLQ's mass $\left(m_{2}\right)$ is not that much restricted from the DM phenomenology or from a vacuum stability point of view as long as $\sin \theta$ remains small. Only for representative purpose, we consider $m_{2}$ to be heavier than $m_{1}$ by $50 \mathrm{GeV}$ and eventually $m_{2}-m_{S}$ remains still below $m_{t}$. The other VLQ's mass [we have a $S U(2)_{L}$ doublet and a singlet VLQs], $\mathcal{M}_{\mathcal{F}}$, falls in between $m_{1}$ and $m_{2}$ and plays an important role in coannihilations among VLQs.
}

While we expect some similarities with respect to DM phenomenology, the study of vacuum stability will be different. For this purpose, we consider the extension of the SM by a scalar singlet DM $S$ along with a vectorlike lepton doublet $E=\left(E^{0}, E^{-}\right)^{T}$ and a neutral singlet lepton $\chi$, all of them being odd under the additional $Z_{2}$ while SM fields are even under the same. The Lagrangian involving the VLLs can then be written as follows:

$$
\begin{aligned}
-\mathcal{L}^{\mathrm{VLL}}= & y_{l_{i j}} \bar{E}_{i} \tilde{H} \chi_{j}+\alpha_{l_{i \beta}} \bar{E}_{R_{i}} S l_{L_{\beta}}+\left(M_{E}\right)_{i j} \bar{E}_{i} E_{j} \\
& +\left(M_{\chi}\right)_{i j} \bar{\chi}_{i} \chi_{j}+\text { H.c. }
\end{aligned}
$$

where $i, j$ are generation indices associated with the additional vectorlike lepton doublets and singlets and $\beta=e, \mu, \tau$. We will specify the required number of generations whenever applicable. Here $E_{i}$ represents the $S U(2)_{L}$ VLL doublets with hypercharge $Y=-\frac{1}{2}$ whereas $\chi_{i}$ represents the $S U(2)_{L}$ VLL singlets with hypercharge $Y=0$. We now proceed with only one generation of VLL doublet and singlet. Thereafter, we discuss implications of the presence of more generations.

After the electroweak symmetry breaking, there will be a mixing among the neutral components of the added VLL doublet and singlet originated due to the presence of the first term in the above Lagrangian. This is similar to the mixing $\theta$ of Sec. II, and here we denote it by $\theta_{l}$ defined by $\tan 2 \theta_{l}=\sqrt{2} y_{l} v /\left(M_{\chi}-M_{E}\right)$, analogous to Eq. (9). The mass eigenvalues $m_{1,2}$ follow the same relation as provided in Eq. (7), replacing $\theta$ by $\theta_{l}, \mathcal{F}$ by $E$ and $f$ by $\chi$. In the scalar sector, the relations Eqs. (2)-(5) remain unaltered.

Since here we will study the scalar singlet DM and vacuum stability in the presence of the VLLs, constraints like stability, perturbativity, relic and DD limits as mentioned in Sec. III are also applicable in this study. Additionally one should consider the constraints coming from the collider searches of the VLLs. The limit from the LEP excludes a singly charged fermion having mass below $100 \mathrm{GeV}$ [61,62], as a result we consider $m_{E^{ \pm}} \geq 100 \mathrm{GeV}$. Due to the presence of the charged VLL, the present setup can also be probed at the LHC. Being a part of the $S U(2)$ doublets, the charged VLLs can be pair produced at the LHC in Drell-Yan processes, $p p \rightarrow$ vectorlike leptons and antilepton pairs, and subsequently undergo Yukawa-driven decays into a DM scalar and a charged SM lepton, $E^{ \pm} \rightarrow$ $S l^{ \pm}$and thus leading to a characteristic opposite sign dilepton plus missing energy $(\not E)$ signature $[63,64]$. In $[65,66]$ which also has a similar setup as ours, the authors have shown that if the mass splitting between the charged and the neutral VLL is less than the mass of the $W^{ \pm}$boson, then $E^{ \pm}$can also decay via the three body suppressed process: $E^{ \pm} \rightarrow E^{0} l^{ \pm} \nu$ giving rise to the displaced vertex signature at LHC.

The additional VLLs in the present setup can also contribute to the electroweak precision test parameters $S$, 


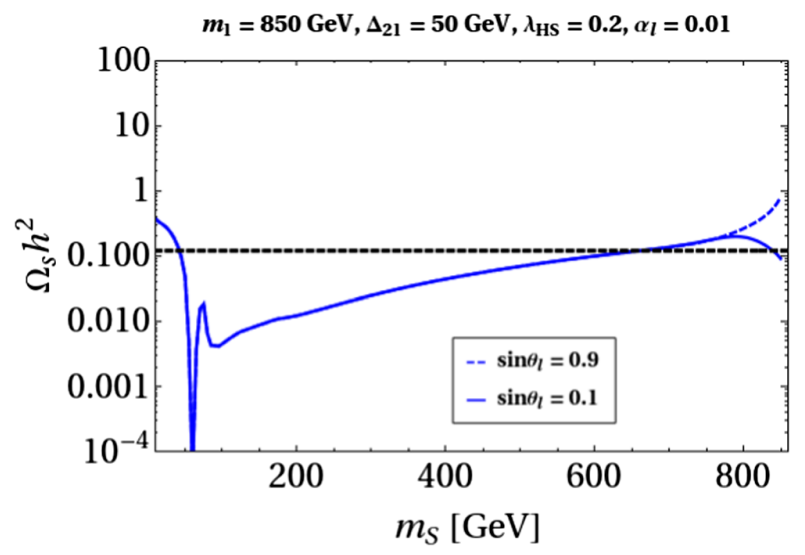

(a)

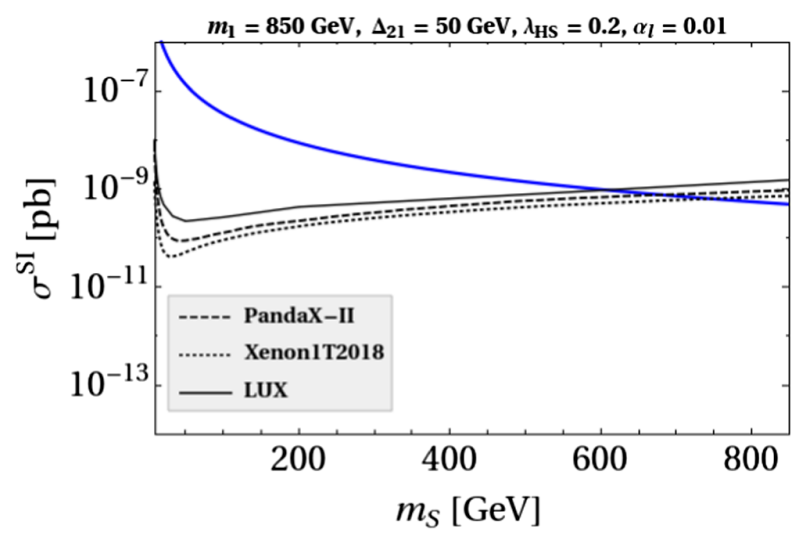

(b)

FIG. 12. Relic density and direct search/XENON1T allowed parameter space of the scalar singlet $(S)$ DM + VLL for different $\sin \theta_{l}$.

$T$ and $U$ [67-69]. The values of these parameters are tightly constrained by experiments. As discussed above, due the presence of doublet and a singlet VLL and their interaction with the SM Higgs (for one generation of VLLs), after the electroweak symmetry breaking three physical states, namely i.e., one charged and two neutral, are obtained. Therefore, the contribution to the precision parameters depends on the masses of the physical states as well as on the mixing angle $\sin \theta_{l}$ in the present setup. One can look at the study in [70] where a detailed analysis of electroweak precision test parameters $S, T$ and $U$ for the setup similar to ours has been presented.

\section{A. DM phenomenology in the presence of VLL}

DM annihilations will proceed through the diagrams similar to the ones mentioned in Figs. 1-3, replacing the VLQ by their VLL counterpart. However as the VLLs do not carry any color quantum number, gluon involved processes would not be present. Other gauge mediated diagrams are present though and continue to play important roles as we will see below.

We provide in the left panel of Fig. 12 the DM relic density $\left(\Omega_{S} h^{2}\right)$ versus mass of the DM $\left(m_{S}\right)$ plot, while parameters like $\Delta_{21} \quad\left(=m_{2}-m_{1}\right)$ and $\alpha_{l}$ (assuming $\left.\alpha_{l_{i \beta}}=\alpha_{l} \delta_{i \beta}\right)$ are fixed at $50 \mathrm{GeV}$ and 0.01 respectively, the same as their respective values in the case of VLQs. However Higgs portal coupling of the DM is kept fixed at an enhanced value, $\lambda_{H S}=0.2$ as compared to its value in the case of VLQ. The requirement of a larger $\lambda_{H S}$ would be evident when we discuss the vacuum stability. Now with such a large $\lambda_{H S}$, the pure singlet scalar DM relic density would be underabundant for DM mass below $600 \mathrm{GeV}$ as evident from the left panel of Fig. 5. Furthermore the presence of VLL having mass suitable for any possible coannihilation would make the situation worsen. Hence we consider a relatively heavy VLL mass set at $850 \mathrm{GeV}$. Note that there is a possibility of satisfying relic by a $\sim 660 \mathrm{GeV}$
DM where no coannihilation involving VLL takes place. Although the relic is satisfied, this particular $m_{S}=$ $660 \mathrm{GeV}$ with $\lambda_{H S}=0.2$ (the very first intersection with correct relic line indicted by the horizontal line) is ruled out due to the associated large DD cross section, unless a heavier DM is considered as seen from the right panel of Fig. 12.

The VLLs start to contribute through coannihilation around $m_{S} \sim 790 \mathrm{GeV}$ and above as indicated by the drop of the relic curve associated with $\sin \theta_{l}=0.1$ in the left panel of Fig. 12 which finally satisfies the relic at $m_{S}=840 \mathrm{GeV}$. This pattern is similar to the one we have obtained in the case with VLQs. Here annihilations of VLLs through gauge mediation play the significant role (almost 80 percent contribution to the relic density of $S$ ). Another interesting pattern is observed for a large $\sin \theta_{l}$. As seen in the left panel of Fig. 12, there is no such change observed with $\sin \theta_{l}=0.9$. It mainly follows the pattern of a regular scalar singlet relic contour plot. Note that with a large $\sin \theta_{l} \sim 0.9$, the lightest VLL with mass $m_{1}$ taking part in the coannihilation and annihilation of VLLs to SM particles consists of mostly the singlet component $\chi$ as compared to its dominant doublet nature in the case with small $\sin \theta_{l}$. Hence gauge mediated processes are naturally suppressed and there is no such effective annihilation of VLL. With large $\theta_{l}$, the only possibility where DM can satisfy the relic and DD cross section is with large $\alpha_{l}$. As the DD cross section involves only the Higgs mediated contribution similar to the one shown in Fig. 4 (which only depends on $\lambda_{H S}$ ), the plot in the right panel is essentially independent of $\sin \theta_{l}$ and $\alpha_{l}$.

\section{B. Vacuum stability in the presence of VLL}

With the above understanding about the relevant parameter space from DM phenomenology, we are now going to analyze the impact of the VLL + scalar singlet setup on vacuum stability. For this purpose, we employ the new set of RG equations provided in Appendix B. In doing 


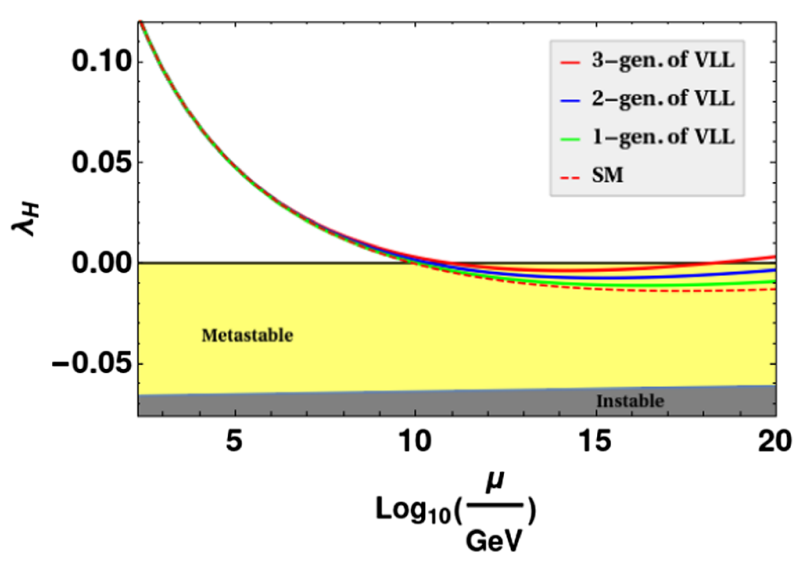

(a)

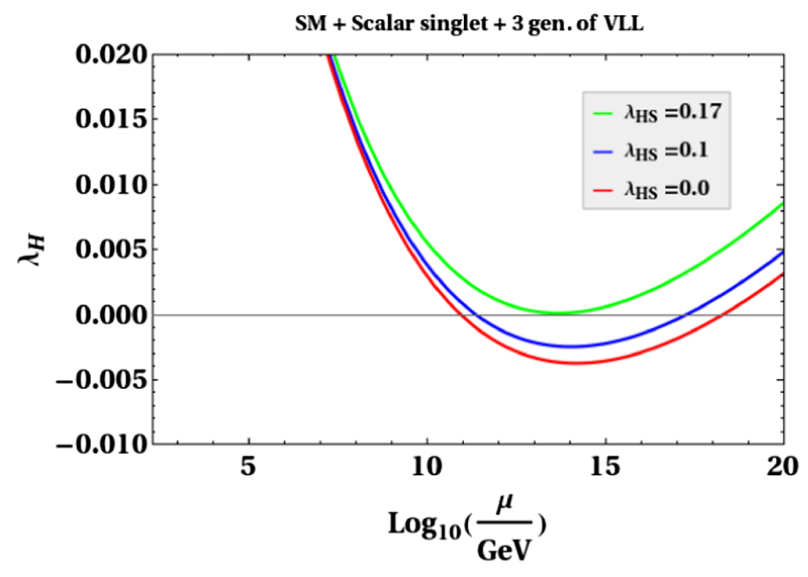

(b)

FIG. 13. (a) Running of Higgs quartic coupling $\lambda_{H}$ against the scale $\mu$ by considering the presence of different generations of vectorlike leptons, while setting $\lambda_{H S}=0$. (b) Running of Higgs quartic coupling for different values of $\lambda_{H S}$ for the three generations of vectorlike leptons.

this analysis, we consider more than one generation of doublet-singlet VLL fermions the reason of which will be clear as we proceed. However for simplicity, we consider $y_{l_{i j}}, \alpha_{l_{i j}}$ as diagonal having equal values $y_{l}$ and $\alpha_{l}$ respectively. Similarly $M_{E}$ and $M_{\chi}$ are taken as diagonal. We maintain the hierarchy (in case of three generations of VLLs) as $\Delta_{31} \gg \Delta_{32} \gg \Delta_{21}$ so that the DM phenomenology remains unaltered and effectively influenced by $m_{1}$ only (i.e., $m_{1}$ only is involved in the effective annihilation cross section of DM). Although the presence of several generations would lead to several mixing angles (involved through the mass matrix including the neutral components of all the VLL singlets and doublets) in general, we assume only one mixing, denoted by $\theta_{l}$, to be effective and ignore other mixings. Following our understanding of the DM analysis, we have fixed certain parameters at their benchmark values as: $\alpha_{l}=0.01, \Delta_{21}=50 \mathrm{GeV}, m_{1}=850 \mathrm{GeV}$, $m_{S}=840 \mathrm{GeV}$ and $\sin \theta_{l}=0.1$. Later we will investigate the variation of $\alpha_{l}, \theta_{l}$ and its impact on the vacuum stability.

In order to understand the impact of VLLs on the running of the Higgs quartic coupling, let us first consider the left panel of Fig. 13. In this plot, we provide running of the SM Higgs quartic coupling $\lambda_{H}$ against the scale $\mu$ considering the presence of different generations of VLL pairs of doublet and singlet, while the presence of the scalar singlet $\mathrm{DM}$ is ignored by setting $\lambda_{H S}=0$. It turns out that, contrary to the case of VLQs, the presence of three generations of VLL doublet and singlet alone cannot keep $\lambda_{H}$ positive at all scales. As we find in the right panel of Fig. 13, a sizable $\lambda_{H S}=0.17$ along with three generations of VLLs (doublet and singlet) can keep the EW vacuum absolutely stable all the way until Planck scale [through the additional contribution in Eq. (24)].

In the right panel of Fig. 13, we notice that $\lambda_{H}$ becomes negative around $10^{11} \mathrm{GeV}$ and again approaches positive before $M_{\mathrm{Pl}}$. Hence the situation with smaller $\lambda_{H S}$, say 0.1 with three generations of VLL, indicates existence of another deeper minimum and EW vacuum is found to be metastable. This is true even for three generations of VLL with $\lambda_{H S}=0$. Note that it requires $\lambda_{H S}$ to be 0.17 in order to make the vacuum absolutely stable. So for the rest of the analysis, where we stress upon the absolute stability only, we consider $\lambda_{H S}=0.2$ and set the DM mass at $840 \mathrm{GeV}$. On the other side, if we accept the metastability of the EW vacuum (with $\lambda_{H S}<$ 0.17 having three-generation VLLs) as a possibility, then a lighterDM mass (along with $m_{1} \gtrsim m_{S}$ ) can also be realized in the present framework.

We now focus on the details of the specific pattern of running of $\lambda_{H}$ in this scenario as compared to the VLQ case. In Fig. 14, $\lambda_{H}$ running is shown in the panel for three different cases: the dashed red line corresponds to the SM variation while dotted and solid lines stand for $\mathrm{SM}+$ scalar singlet DM (with $\lambda_{H S}=0.2$ ) and $\mathrm{SM}+$ scalar singlet $\mathrm{DM}+3$ generations of VLLs. In the right panel, running of the gauge couplings $g_{1,2,3}$ is shown. We notice that while $g_{3}$ remains unaffected, $g_{1}$ and $g_{2}$ undergo a positive shift (compared to the SM running) due to the presence of VLL. These shifts can be understood by looking at the additional contributions to the respective $\beta$ functions as in Eq. (22) as

$$
\begin{gathered}
\beta g_{1}=\beta_{g_{1}}^{\mathrm{SM}}+\beta_{g_{1}}^{\mathrm{VLL}(1)}=\beta_{g_{1}}^{\mathrm{SM}}+\frac{g_{1}^{3}}{16 \pi^{2}}\left[\frac{4}{5}\left(2 n_{2} Y_{E}^{2}+n_{1} Y_{\chi}^{2}\right)\right] \\
\beta g_{2}=\beta_{g_{2}}^{\mathrm{SM}}+\beta_{g_{2}}^{\mathrm{VLL}(1)}=\beta_{g_{2}}^{\mathrm{SM}}+\frac{g_{2}^{3}}{16 \pi^{2}}\left(\frac{2}{3} n_{2}\right),
\end{gathered}
$$

where $n_{2}$ is the number of $S U(2)$ doublets, $n_{1}$ is the number of $S U(2)$ singlets, and $Y_{E, \chi}$ are the hypercharges of $E$ and $\chi$ 


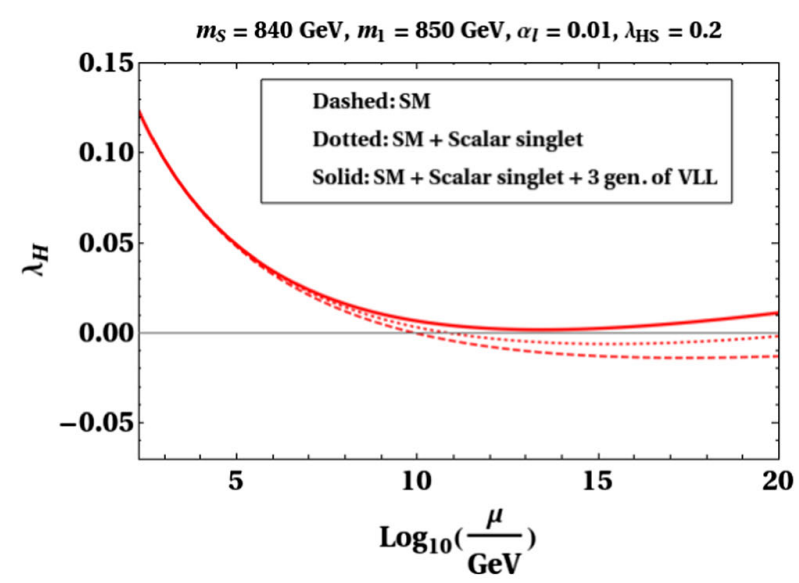

(a)

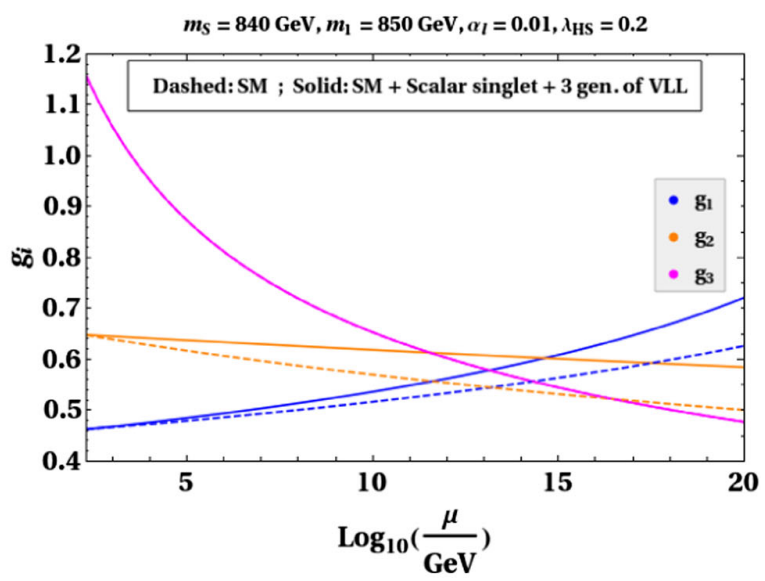

(b)

FIG. 14. (a) Running of Higgs quartic coupling $\lambda_{H}$ against the scale $\mu$ for the SM, SM + scalar singlet and $\mathrm{SM}+$ scalar singlet +3 generation of VLLs scenario. (b) Running of gauge couplings against the scale $\mu$ for the SM and SM + scalar singlet +3 generations of VLLs scenario.

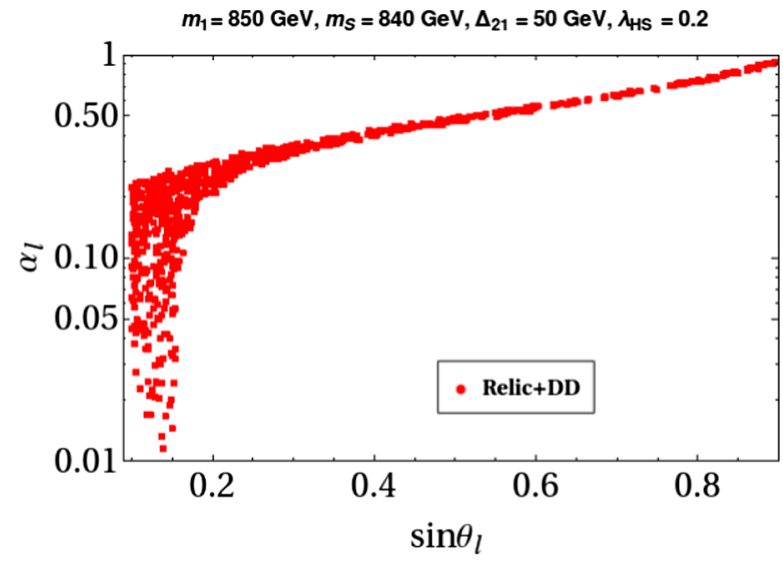

(a)

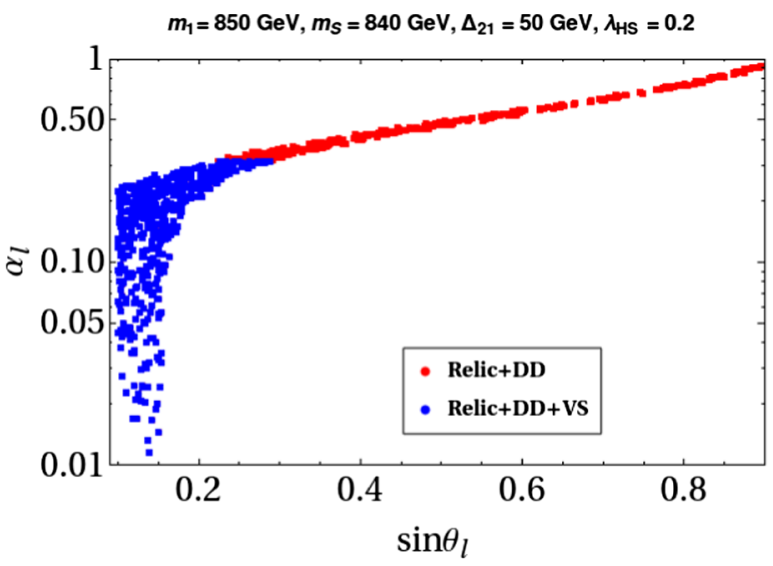

(b)

FIG. 15. Contour plot showing allowed parameter space from (a) the DM constraints, (b) DM and the vacuum stability constraints in the $\alpha_{l}-\sin \theta_{l}$ plane.

respectively. ${ }^{2}$ Though this helps in decreasing the $y_{t}$ value due to running, it is not adequate to keep $\lambda_{H}$ positive at any scale beyond $\Lambda_{I}^{\mathrm{SM}}$ even with $\mathrm{SM}+$ three generations of VLLs. In lifting the $\lambda_{H}$ to the positive side for any scale until $M_{\mathrm{Pl}}$ or to maintain the absolute stability, contributions of both $\lambda_{H S}$ [through the additional term in $\beta_{\lambda_{H}}$ similar to Eq. (24)] and three generations of VLL [due to the presence of $n_{1}, n_{2}$ in $\beta_{g_{1}, g_{2}}$ via Eq. (22)] turn out to be important.

\section{Combined analysis of DM and EW vacuum stability}

In this section, we consider the effect of varying the two parameters $\alpha_{l}$ and $\theta_{l}$. Therefore we perform a scan over a

\footnotetext{
${ }^{2}$ Although here we represent the one-loop beta functions for the BSM part for discussion purposes, we finally include the twoloop beta functions while studying the running.
}

wide range of $\alpha_{l}$ and $\theta_{l}$ and represent the correlation plot obtained in the left panel of Fig. 15. These points in red correspond to the correct DM relic density and simultaneously they satisfy the DD constraints. In generating the plot, we fix $m_{S}=840 \mathrm{GeV}, m_{1}=850 \mathrm{GeV}, \Delta_{21}=$ $50 \mathrm{GeV}$ and $\lambda_{H S}=0.2$. We find that for larger $\sin \theta_{l}$, only large values of $\alpha_{l}$ are allowed. Note that contrary to the VLQ case, $\alpha_{l}$ does not participate in the DD cross section as the associated interaction involves leptons only. Hence from the DD point of view, no restriction can be imposed on $\alpha_{l}$. The criteria for satisfying relic density, as we have seen in DM subsection is that the annihilation of VLLs via gauge mediation can contribute to the required DM annihilation cross section in small mixing angle limits. Now for large $\theta_{l}$, as we have already pointed out in Sec. VII A in the context of Fig. 12, a relatively large mixing angle $\sim 0.9$ (indicating $m_{1}$ is mostly made up of 
singlet VLL) is disallowed as it will not correspond to sufficient annihilation of this type (gauge mediated processes) required to satisfy the relic. However the situation changes when $\alpha_{l}$ is also large. This is due to the fact that coannihilation processes of DM involving large $\alpha_{l}$ can provide the required relic.

In the right panel of Fig. 15, we introduce the constraints from vacuum stability. The blue portion now denotes the allowed region of parameter space in the $\alpha_{l}-\theta_{l}$ plane, while the red portion is disallowed from vacuum stability constraints, in particular due to the violation of copositivity criteria of Eq. (11). In this context, it can be noted that terms proportional to $\alpha^{4}$ present in $\beta_{\lambda_{S}}$ (see Appendix B for its expression) make $\lambda_{S}$ negative at some high scale. Hence applying the copositivity conditions, the restricted parameter space for $\alpha_{l}-\theta_{l}$ is obtained.

\section{DISCUSSION AND CONCLUSION}

We have studied the possibility of sub-TeV scalar singlet dark matter along with electroweak vacuum stability by incorporating the presence of additional vectorlike fermions. While a pure scalar singlet DM extension of the standard model can satisfy the requirement of correct DM phenomenology along with EW vacuum stability only for $\mathrm{DM}$ mass above a $\mathrm{TeV}$, introduction of additional vectorlike fermions is shown to bring it down to the sub$\mathrm{TeV}$ regime. Due to the presence of new coannihilation channels between DM and vectorlike fermions (most importantly the annihilations of VLF), it is possible to satisfy DM relic criteria without getting into conflict with the direct detection data. The same additional fermions also play an instrumental role in keeping the Higgs quartic coupling positive at all scales up to $M_{\mathrm{Pl}}$ dominantly through their contributions to the RG evolution of SM gauge couplings.

Similar to the extension with VLQ, the VLL extension also gives rise to additional annihilation and coannihilation channels of DM, as can be seen from the interaction terms included in the Lagrangian. One advantage of this extension compared to the VLQ extension discussed above is that, it does not give rise to an additional contribution to DM-nucleon scattering at the radiative or one-loop level. Also, the additional contribution to the DM relic from Sommerfeld enhancement is absent in this scenario. While one can still have sub-TeV scalar singlet DM allowed from all relevant constraints, the criteria of absolute $\mathrm{EW}$ vacuum stability all the way until Planck scale requires at least three generations of such additional leptons along with a sizable contribution from the DM portal coupling with the SM Higgs. This is precisely due to the difference in the way VLQs contribute to RG running of $S U(3)$ gauge coupling from the way VLLs contribute to $S U(2)$ (dominantly) gauge coupling where the latter do not have any additional color degrees of freedom.

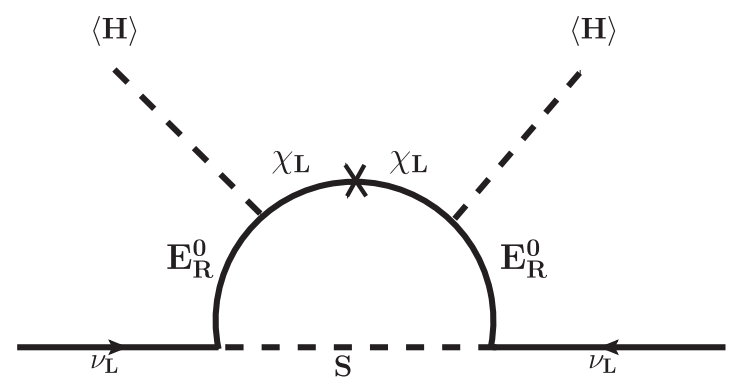

FIG. 16. $\quad \nu$-mass generation at one loop.

While the construction with VLL may appear nonminimal compared to the VLQ extension, the additional three families of leptons can play a nontrivial role in generating light neutrino masses at the one-loop level as shown in Fig. 16 [71-73]. This however requires the addition of Majorana mass terms for the $\chi$ field, which are in fact allowed by the symmetry of the model. A similar construction has been exercised recently in [74] in the presence of Majorana masses for both $\chi_{L}$ and $\chi_{R}$. Since the very details of neutrino mass expression do not carry a direct connection with the DM and vacuum stability part of our scenario, we refrain from studying the details of neutrino mass here.

We have studied the two extensions of the scalar singlet DM model by adopting a minimalistic approach. While our studies have been motivated primarily by the possibility of sub-TeV DM and EW vacuum stability, the scenarios can offer very rich phenomenology in terms of collider physics, indirect detection of dark matter, charged lepton flavor violation etc. While one can get the additional contribution to dijet plus missing energy or dilepton plus missing energy signatures due to vectorlike quarks and leptons respectively, the same additional fermions can also boost DM annihilation to gamma rays, a tantalizing indirect detection signature that has been searched for at several experiments. The leptonic extension of the model which also generates light neutrino masses at one loop can in principle give rise to observable charged lepton flavor violation like $\mu \rightarrow e \gamma$, $\mu \rightarrow 3 e$ etc., especially in the regime where singlet DM has order one Yukawa coupling with the additional fermions. Another possible direction is to consider a UV completion of this minimal model where $Z_{2}$ symmetry can be embedded in a gauge symmetry which also predicts the scale of additional vectorlike fermions. We leave such aspects of our current scenario to future works.

\section{ACKNOWLEDGMENTS}

D. B. acknowledges the support from Early Career Research Award from DST-SERB, Government of India (Reference No. ECR/2017/001873). R. R. would like to thank Basabendu Barman, Abhijit Kumar Saha, Drona Vatsyayan and Lopamudra Mukherjee for various useful discussions during the course of this work. 


\section{APPENDIX A: ONE-LOOP $\beta$ FUNCTIONS FOR VLQ SCENARIO}

Below we provide the one-loop $\beta$ functions for all the couplings involved in the vectorlike quark scenario. While generating the $\beta$ functions we have considered one complete family of VLQ, i.e., one double and one singlet. In the expressions below color charge $N_{c}=3$ for all the VLQs, $n_{1}$ and $n_{2}$ represent the number of $S U(2)$ singlet and doublet VLLs respectively while $n_{3}$ corresponds to the number of $S U$ (3) triplet vectorlike fields and finally $n_{F}$ represents the number of complete VLQ families coupled to the SM Higgs. Due to the involvement of one complete family of VLQs all $n_{1}, n_{2}, n_{F}$ are 1 whereas $n_{3}=3$. Here, $Y_{\mathcal{F}}=-\frac{1}{6}$ and $Y_{f^{\prime}}=\frac{2}{3}$ are the hypercharges of $\mathcal{F}$ and $f^{\prime}$ respectively.

\section{SM couplings}

$$
\begin{gathered}
\beta_{g_{1}}=\beta_{g_{1}}^{\mathrm{SM}}+\beta_{g_{1}}^{\mathrm{VLQ}}=\beta_{g_{1}}^{\mathrm{SM}}+\frac{g_{1}^{3}}{16 \pi^{2}}\left(\frac{4}{5} N_{c}\left(2 n_{2} Y_{\mathcal{F}}^{2}+n_{1} Y_{f^{\prime}}^{2}\right)\right)=\beta_{g_{1}}^{\mathrm{SM}}+\frac{1}{16 \pi^{2}}\left(\frac{6}{5} g_{1}^{3}\right) \\
\beta_{g_{2}}=\beta_{g_{2}}^{\mathrm{SM}}+\beta_{g_{2}}^{\mathrm{VLQ}}=\beta_{g_{2}}^{\mathrm{SM}}+\frac{g_{2}^{3}}{16 \pi^{2}}\left(\frac{2}{3} N_{c} n_{2}\right)=\beta_{g_{2}}^{\mathrm{SM}}+\frac{1}{16 \pi^{2}}\left(2 g_{2}^{3}\right) \\
\beta_{g_{3}}=\beta_{g_{3}}^{\mathrm{SM}}+\beta_{g_{3}}^{\mathrm{VLQ}}=\beta_{g_{3}}^{\mathrm{SM}}+\frac{g_{3}^{3}}{16 \pi^{2}}\left(\frac{2 n_{3}}{3}\right)=\beta_{g_{3}}^{\mathrm{SM}}+\frac{1}{16 \pi^{2}}\left(2 g_{3}^{3}\right) \\
\beta_{\lambda_{H}}=\beta_{\lambda_{H}}^{\mathrm{SM}}+\beta_{\lambda_{H}}^{\mathrm{S}}+\beta_{\lambda_{H}}^{\mathrm{VLQ}}=\beta_{\lambda_{H}}^{\mathrm{SM}}+\beta_{\lambda_{H}}^{\mathrm{S}}+\frac{2 n_{F}}{16 \pi^{2}}\left(4 N_{c} y_{l} \lambda_{H}-2 N_{c} y^{4}\right)=\beta_{\lambda_{H}}^{\mathrm{SM}}+\frac{1}{16 \pi^{2}}\left(\frac{1}{2} \lambda_{H S}^{2}-12 y^{4}+24 y^{2} \lambda_{H}\right) \\
\beta_{y_{t}}=\beta_{y_{t}}^{\mathrm{SM}}+\beta_{y_{t}}^{\mathrm{VLQ}}=\beta_{y_{t}}^{\mathrm{SM}}+\frac{n_{F} y_{t}}{16 \pi^{2}}\left(2 N_{c} y^{2}\right) \quad \beta_{y_{t}}^{\mathrm{SM}}+\frac{1}{16 \pi^{2}}\left(6 y_{t} y^{2}\right) .
\end{gathered}
$$

\section{BSM couplings}

$$
\begin{gathered}
\beta_{\lambda_{S}}^{(1)}=\frac{1}{16 \pi^{2}}\left(3\left(16 \lambda_{S} \alpha_{1}^{2}-48 \alpha_{2}^{4}+4 \lambda_{H S}^{2}+8 \lambda_{S} \alpha_{2}^{2}-96 \alpha_{1}^{4}+\lambda_{S}^{2}\right)\right) \\
\beta_{\lambda_{H S}(1)}^{(1)}=\frac{1}{16 \pi^{2}}\left(-\frac{9}{10} g_{1}^{2} \lambda_{H S}-\frac{9}{2} g_{2}^{2} \lambda_{H S}+4 \lambda_{H S}^{2}+\lambda_{H S} \lambda_{S}+12 \lambda_{H S} y^{2}+12 \lambda_{H S} \lambda\right. \\
+24\left(-y^{2}+\lambda_{H S}\right) \alpha_{1}^{2}+24 y \alpha_{1} y_{t} \alpha_{2}+24 y \alpha_{1} y_{t} \alpha_{2}-24 \alpha_{1}^{2} y_{t}^{2}+12 \lambda_{H S} \alpha_{2}^{2} \\
\left.-24 y^{2} \alpha_{2}^{2}-24 y_{t}^{2} \alpha_{2}^{2}+6 \lambda_{H S} y_{t}^{2}\right) \\
\beta_{\alpha_{1}}^{(1)}=\frac{1}{16 \pi^{2}}\left(\frac{1}{10}\left(5\left(-4 y y_{t} \alpha_{2}+y_{t}^{2} \alpha_{1}\right)-\alpha_{1}\left(-150 \alpha_{1}^{2}+45 g_{2}^{2}-5 y^{2}-60 \alpha_{2}^{2}+80 g_{3}^{2}+g_{1}^{2}\right)\right)\right) \\
\beta_{\alpha_{2}}^{(1)}=\frac{1}{16 \pi^{2}}\left(-4 y y_{t} \alpha_{1}+\alpha_{2}\left(12 \alpha_{1}^{2}-8 g_{3}^{2}+9 \alpha_{2}^{2}-\frac{8}{5} g_{1}^{2}+y^{2}\right)+y_{t}^{2} \alpha_{2}\right) .
\end{gathered}
$$




\section{APPENDIX B: ONE-LOOP $\beta$ FUNCTIONS FOR VLL SCENARIO (THREE GENERATIONS)}

Here, we provide the one-loop $\beta$ functions of all the relevant couplings by considering three complete generations of VLLs, i.e., three doublets and three singlets. In the expressions below color charge $N_{c}=1$ for all the VLLs, $n_{1}, n_{2}, n_{F}$ are 3 due to the presence of three complete generations of VLL families whereas $n_{3}=0$ and finally $Y_{E}=-\frac{1}{2}$ and $Y_{\chi}=0$ are the hypercharges of $E$ and $\chi$. Here we have also assumed that $y_{l_{i j}}, \alpha_{l_{i j}}$ are diagonal having equal values $y_{l}$ and $\alpha_{l}$ respectively.

$$
\begin{aligned}
& \text { 1. SM couplings } \\
& \beta_{g_{1}}=\beta_{g_{1}}^{\mathrm{SM}}+\beta_{g_{1}}^{\mathrm{VLL}}=\beta_{g_{1}}^{\mathrm{SM}}+\frac{g_{1}^{3}}{16 \pi^{2}}\left(\frac{4}{5} N_{c}\left(2 n_{2} Y_{E}^{2}+n_{1} Y_{\chi}^{2}\right)\right)=\beta_{g_{1}}^{\mathrm{SM}}+\frac{1}{16 \pi^{2}}\left(\frac{6}{5} g_{1}^{3}\right) \\
& \beta_{g_{2}}=\beta_{g_{2}}^{\mathrm{SM}}+\beta_{g_{2}}^{\mathrm{VLL}}=\beta_{g_{2}}^{\mathrm{SM}}+\frac{g_{2}^{3}}{16 \pi^{2}}\left(\frac{2}{3} N_{c} n_{2}\right)=\beta_{g_{2}}^{\mathrm{SM}}+\frac{1}{16 \pi^{2}}\left(2 g_{2}^{3}\right) \\
& \beta_{g_{3}}=\beta_{g_{3}}^{\mathrm{SM}}+\beta_{g_{3}}^{\mathrm{VLL}}=\beta_{g_{3}}^{\mathrm{SM}}+\frac{g_{3}^{3}}{16 \pi^{2}}\left(\frac{2 n_{3}}{3}\right)=\beta_{g_{3}}^{\mathrm{SM}} \\
& \beta_{\lambda_{H}}=\beta_{\lambda_{H}}^{\mathrm{SM}}+\beta_{\lambda_{H}}^{\mathrm{S}}+\beta_{\lambda_{H}}^{\mathrm{VLL}}=\beta_{\lambda_{H}}^{\mathrm{SM}}+\beta_{\lambda_{H}}^{\mathrm{S}}+\frac{2 n_{F}}{16 \pi^{2}}\left(4 N_{c} y_{l} \lambda_{H}-2 N_{c} y_{l}^{4}\right)=\beta_{\lambda_{H}}^{\mathrm{SM}}+\frac{1}{16 \pi^{2}}\left(\frac{1}{2} \lambda_{H S}^{2}+24 \lambda_{H} y_{l}^{2}-12 y_{l}^{4}\right) \\
& \beta_{y_{t}}=\beta_{y_{t}}^{\mathrm{SM}}+\beta_{y_{t}}^{\mathrm{VLL}}=\beta_{y_{t}}^{\mathrm{SM}}+\frac{n_{F} y_{t}}{16 \pi^{2}}\left(2 N_{c} y_{l}^{2}\right) \quad \beta_{y_{t}}^{\mathrm{SM}}+\frac{1}{16 \pi^{2}}\left(6 y_{t} y_{l}^{2}\right) .
\end{aligned}
$$

\section{BSM couplings}$$
\beta_{\lambda_{S}}=\frac{1}{16 \pi^{2}}\left(48 \lambda_{S} \alpha_{l}-288 \alpha_{l}^{4}+3\left(4 \lambda_{H S}^{2}+\lambda_{S}^{2}\right)\right)
$$$$
\beta_{\lambda_{H S}}=\frac{1}{16 \pi^{2}}\left(-\frac{9}{10} g_{1}^{2} \lambda_{H S}-\frac{9}{2} g_{2}^{2} \lambda_{H S}+4 \lambda_{H S}^{2}+\lambda_{H S} \lambda_{S}+12 \lambda_{H S} \lambda+12 \lambda_{H S} y_{l}^{2}+24 \lambda_{H S} \alpha_{l}^{2}+6 \lambda_{H S} y_{t}^{2}-24 y_{l}^{2} \alpha_{l}^{2}\right)
$$$$
\beta_{y_{l}}=\frac{1}{16 \pi^{2}}\left(\frac{15}{2} y_{l}^{3}+y_{l}\left(3 y_{t}^{2}-\frac{9}{20} g_{1}^{2}-\frac{9}{4} g_{2}^{2}\right)\right)
$$$$
\beta_{\alpha_{1}}=\frac{1}{16 \pi^{2}}\left(\frac{1}{10}\left(5 y_{l}^{2} \alpha_{l}+150 \alpha_{l}^{3}-9 \alpha_{l}\left(5 g_{2}^{2}+g_{1}^{2}\right)\right)\right) \text {. }
$$

[1] G. Hinshaw et al. (WMAP Collaboration), Astrophys. J. Suppl. Ser. 208, 19 (2013).

[2] N. Aghanim et al. (Planck Collaboration), Astron. Astrophys. 641, A6 (2020).

[3] E. W. Kolb and M. S. Turner, Front. Phys. 69, 1 (1990).

[4] V. Silveira and A. Zee, Phys. Lett. 161B, 136 (1985).

[5] J. McDonald, Phys. Rev. D 50, 3637 (1994).

[6] C. P. Burgess, M. Pospelov, and T. ter Veldhuis, Nucl. Phys. B619, 709 (2001).
[7] P. Athron et al. (GAMBIT Collaboration), Eur. Phys. J. C 77, 568 (2017).

[8] D. S. Akerib et al. (LUX Collaboration), Phys. Rev. Lett. 118, 021303 (2017).

[9] A. Tan et al. (PandaX-II Collaboration), Phys. Rev. Lett. 117, 121303 (2016).

[10] X. Cui et al. (PandaX-II Collaboration), Phys. Rev. Lett. 119, 181302 (2017).

[11] E. Aprile et al. (XENON Collaboration), Phys. Rev. Lett. 119, 181301 (2017). 
[12] E. Aprile et al. (XENON Collaboration), Phys. Rev. Lett. 121, 111302 (2018).

[13] ATLAS Collaboration, Search for invisible Higgs boson decays with vector boson fusion signatures with the ATLAS detector using an integrated luminosity of $139 \mathrm{fb}^{-1}$, Report No. ATLAS-CONF-2020-008, 2020.

[14] I. Garg, S. Goswami, K. Vishnudath, and N. Khan, Phys. Rev. D 96, 055020 (2017).

[15] G. Isidori, G. Ridolfi, and A. Strumia, Nucl. Phys. B609, 387 (2001).

[16] E. Greenwood, E. Halstead, R. Poltis, and D. Stojkovic, Phys. Rev. D 79, 103003 (2009).

[17] J. Ellis, J. R. Espinosa, G. F. Giudice, A. Hoecker, and A. Riotto, Phys. Lett. B 679, 369 (2009).

[18] J. Elias-Miro, J. R. Espinosa, G. F. Giudice, G. Isidori, A. Riotto, and A. Strumia, Phys. Lett. B 709, 222 (2012).

[19] S. Alekhin, A. Djouadi, and S. Moch, Phys. Lett. B 716, 214 (2012).

[20] D. Buttazzo, G. Degrassi, P. P. Giardino, G. F. Giudice, F. Sala, A. Salvio, and A. Strumia, J. High Energy Phys. 12 (2013) 089.

[21] L. A. Anchordoqui, I. Antoniadis, H. Goldberg, X. Huang, D. Lust, T. R. Taylor, and B. Vlcek, J. High Energy Phys. 02 (2013) 074.

[22] Y. Tang, Mod. Phys. Lett. A 28, 1330002 (2013).

[23] A. Salvio, Phys. Lett. B 743, 428 (2015).

[24] A. Salvio, Phys. Rev. D 99, 015037 (2019).

[25] A. K. Saha and A. Sil, Phys. Lett. B 765, 244 (2017).

[26] T. Toma, Phys. Rev. Lett. 111, 091301 (2013).

[27] F. Giacchino, L. Lopez-Honorez, and M. H. Tytgat, J. Cosmol. Astropart. Phys. 10 (2013) 025.

[28] F. Giacchino, L. Lopez-Honorez, and M. H. Tytgat, J. Cosmol. Astropart. Phys. 08 (2014) 046.

[29] A. Ibarra, T. Toma, M. Totzauer, and S. Wild, Phys. Rev. D 90, 043526 (2014).

[30] B. Barman, S. Bhattacharya, P. Ghosh, S. Kadam, and N. Sahu, Phys. Rev. D 100, 015027 (2019).

[31] B. Barman, D. Borah, P. Ghosh, and A. K. Saha, J. High Energy Phys. 10 (2019) 275.

[32] B. Barman, A. Dutta Banik, and A. Paul, Phys. Rev. D 101, 055028 (2020).

[33] F. Giacchino, A. Ibarra, L. Lopez Honorez, M. H. G. Tytgat, and S. Wild, J. Cosmol. Astropart. Phys. 02 (2016) 002.

[34] S. Baek, P. Ko, and P. Wu, J. High Energy Phys. 10 (2016) 117.

[35] S. Baek, P. Ko, and P. Wu, J. Cosmol. Astropart. Phys. 07 (2018) 008.

[36] S. Colucci, B. Fuks, F. Giacchino, L. Lopez Honorez, M. H. G. Tytgat, and J. Vandecasteele, Phys. Rev. D 98, 035002 (2018).

[37] S. Biondini and S. Vogl, J. High Energy Phys. 11 (2019) 147.

[38] P. Ghosh, A. K. Saha, and A. Sil, Phys. Rev. D 97, 075034 (2018).

[39] S. Gopalakrishna and A. Velusamy, Phys. Rev. D 99, 115020 (2019).

[40] J. Horejsi and M. Kladiva, Eur. Phys. J. C 46, 81 (2006).

[41] G. Bhattacharyya and D. Das, Pramana 87, 40 (2016).

[42] A. Buckley, J. Butterworth, L. Corpe, D. Huang, and P. Sun, arXiv:2006.07172.

[43] G. Abbiendi et al. (OPAL Collaboration), Phys. Lett. B 545, 272 (2002); 548, 258(E) (2002).
[44] D. Barducci, G. Belanger, J. Bernon, F. Boudjema, J. Da Silva, S. Kraml, U. Laa, and A. Pukhov, Comput. Phys. Commun. 222, 327 (2018).

[45] S. Bhattacharya, P. Ghosh, A. K. Saha, and A. Sil, J. High Energy Phys. 03 (2020) 090.

[46] A. Dutta Banik, R. Roshan, and A. Sil, arXiv:2009.01262.

[47] G. Degrassi, S. Di Vita, J. Elias-Miro, J. R. Espinosa, G. F. Giudice, G. Isidori, and A. Strumia, J. High Energy Phys. 08 (2012) 098.

[48] N. Haba, K. Kaneta, and R. Takahashi, J. High Energy Phys. 04 (2014) 029.

[49] N. Khan and S. Rakshit, Phys. Rev. D 90, 113008 (2014).

[50] V. V. Khoze, C. McCabe, and G. Ro, J. High Energy Phys. 08 (2014) 026.

[51] M. Gonderinger, Y. Li, H. Patel, and M. J. Ramsey-Musolf, J. High Energy Phys. 01 (2010) 053.

[52] M. Gonderinger, H. Lim, and M. J. Ramsey-Musolf, Phys. Rev. D 86, 043511 (2012).

[53] W. Chao, M. Gonderinger, and M. J. Ramsey-Musolf, Phys. Rev. D 86, 113017 (2012).

[54] E. Gabrielli, M. Heikinheimo, K. Kannike, A. Racioppi, M. Raidal, and C. Spethmann, Phys. Rev. D 89, 015017 (2014).

[55] A. Dutta Banik, A. K. Saha, and A. Sil, Phys. Rev. D 98, 075013 (2018).

[56] F. Staub, Comput. Phys. Commun. 185, 1773 (2014).

[57] A. De Simone, G. F. Giudice, and A. Strumia, J. High Energy Phys. 06 (2014) 081.

[58] S. El Hedri, A. Kaminska, M. de Vries, and J. Zurita, J. High Energy Phys. 04 (2017) 118.

[59] A. Mitridate, M. Redi, J. Smirnov, and A. Strumia, J. Cosmol. Astropart. Phys. 05 (2017) 006.

[60] S. Biondini and M. Laine, J. High Energy Phys. 04 (2018) 072.

[61] P. Achard et al. (L3 Collaboration), Phys. Lett. B 517, 75 (2001).

[62] J. Abdallah et al. (DELPHI Collaboration), Eur. Phys. J. C 31, 421 (2003).

[63] K. Kowalska and E. M. Sessolo, J. High Energy Phys. 09 (2017) 112.

[64] S. Chakraborti and R. Islam, Phys. Rev. D 101, 115034 (2020).

[65] S. Bhattacharya, N. Sahoo, and N. Sahu, Phys. Rev. D 96, 035010 (2017).

[66] S. Bhattacharya, B. Karmakar, N. Sahu, and A. Sil, J. High Energy Phys. 05 (2017) 068.

[67] M. E. Peskin and T. Takeuchi, Phys. Rev. D 46, 381 (1992).

[68] F. del Aguila, J. de Blas, and M. Perez-Victoria, Phys. Rev. D 78, 013010 (2008).

[69] J. Erler and P. Langacker, Phys. Rev. Lett. 105, 031801 (2010).

[70] S. Bhattacharya, P. Ghosh, N. Sahoo, and N. Sahu, Front. Phys. 7, 80 (2019).

[71] A. G. Dias, C. A. de S. Pires, P. S. Rodrigues da Silva, and A. Sampieri, Phys. Rev. D 86, 035007 (2012).

[72] S. Esch, M. Klasen, D. R. Lamprea, and C. E. Yaguna, Eur. Phys. J. C 78, 88 (2018).

[73] S. May, Institut für Theoretische Physik, Westfälische Wilhelms-Universität Münster, Ph.D. thesis, 2020 [arXiv:2003.04157].

[74] P. Konar, A. Mukherjee, A. K. Saha, and S. Show, Phys. Rev. D 102, 015024 (2020). 